\title{
Adjoint-based error estimation and adaptive mesh refinement for the RANS and $k$ - $\omega$ turbulence model equations
}

\author{
Ralf Hartmann ${ }^{\mathrm{a}, \mathrm{b}, *}$, Joachim Held ${ }^{\mathrm{a}, \mathrm{b}}$, Tobias Leicht ${ }^{\mathrm{a}, \mathrm{b}}$ \\ ${ }^{a}$ Institute of Aerodynamics and Flow Technology, DLR (German Aerospace Center), Lilienthalplatz 7, 38108 Braunschweig, Germany \\ ${ }^{b}$ Institute of Scientific Computing, TU Braunschweig, Germany
}

\begin{abstract}
In this article we present the extension of the a posteriori error estimation and goal-oriented mesh refinement approach from laminar to turbulent flows, which are governed by the Reynolds-averaged Navier-Stokes and $k$ - $\omega$ turbulence model (RANS- $k \omega$ ) equations. In particular, we consider a discontinuous Galerkin discretization of the RANS- $k \omega$ equations and use it within an adjoint-based error estimation and adaptive mesh refinement algorithm that targets the reduction of the discretization error in single as well as in multiple aerodynamic force coefficients. The accuracy of the error estimation and the performance of the goal-oriented mesh refinement algorithm is demonstrated for various test cases, including a two-dimensional turbulent flow around a three-element high lift configuration and a threedimensional turbulent flow around a wing-body configuration.
\end{abstract}

Keywords: Error estimation, goal-oriented mesh refinement, turbulent flows, discontinuous Galerkin method

\section{Introduction}

The past few years have seen considerable progress in the development of higher order and adaptive discontinuous Galerkin (DG) methods for aerodynamic flows $[9,1,2,3,4,7,5,10,6,8]$. For example, the European research project ADIGMA [11] concentrated the effort of European scientists on the development of adaptive higher-order variational methods for aerospace applications. In this work we present the methods and algorithms developed in this project for an efficient and reliable prediction of aerodynamic force coefficients for two-dimensional (2d) and three-dimensional (3d) turbulent flows.

Aerodynamic force coefficients, such as the drag, lift and moment coefficients, are important quantities in aerodynamic flow simulations. In addition to the accurate approximation of these quantities it is of increasing importance, particularly in the field of uncertainty quantification, to estimate the error in the computed quantities. By employing a duality argument, estimates can be derived for the error measured in terms of an aerodynamic force coefficient. The error estimate includes primal residuals multiplied by the solution to an adjoint problem that is related to the force coefficient. The error estimate can be decomposed into a sum of local adjoint-based indicators which can drive a goal-oriented adaptive mesh refinement algorithm specifically tailored to the accurate and efficient approximation of the aerodynamic force coefficient under consideration.

The approach of error estimation and goal-oriented mesh refinement for specific target quantities has been developed in $[12,13]$. It has been transferred to compressible flows in the context of discontinuous Galerkin methods in $[14,15]$ for inviscid flows and extended in $[16,17,18]$ to viscous laminar flows; we refer to [19] for related work based on finite volume methods. Subsequently, this approach has been combined with anisotropic hierarchic refinement for laminar compressible flows in $[20,21,22]$ and with a regeneration of output-adapted meshes using anisotropic mesh metrics in [23], see also [26, 24, 25] for related work. Furthermore, the adjoint-based error estimation and mesh refinement approach has been extended from single to multiple target quantities in $[27,28]$. Usually being based on

\footnotetext{
${ }^{*}$ Corresponding author Leicht)

Email addresses: Ralf .Hartmann@dlr.de (Ralf Hartmann), Joachim.Held@dlr.de (Joachim Held), Tobias.Leicht@dlr.de (Tobias
} 
body-aligned regular meshes, the adjoint-based mesh refinement has also been applied to embedded-boundary Cartesian meshes [29, 30], and it has been extended to the simplex cut-cell approach in [3]. It has been extended to $2 \mathrm{~d}$ turbulent flows, which are governed by the RANS and Spalart-Allmaras equations in [23].

In this article we extend the approach of adjoint-based error estimation and adaptive mesh refinement in several aspects. We present it for the discontinuous Galerkin discretization of turbulent flows as governed by the Reynoldsaveraged Navier-Stokes and $k-\omega$ turbulence model equations. We demonstrate it for aerodynamic flow problems, including a turbulent flow around the L1T2 three-element high-lift configuration and a turbulent flow around the DLR-F6 wing-body configuration. Furthermore, we combine it with the approach of adjoint-based error estimation and adaptive mesh refinement for multiple force coefficients as previously developed for $2 \mathrm{~d}$ laminar flows in [28].

This article is structured as follows: In Sections 2 and 3 we recall the RANS- $k \omega$ equations and give reference to the discontinuous Galerkin discretization employed. Then, in Section 4 we recall the approach of adjoint-based error estimation and goal-oriented mesh refinement that targets single and multiple aerodynamic force coefficients. Then, in Section 5, the main section of this work, we demonstrate the performance and accuracy of these methods and algorithms for three aerodynamic test cases of increasing complexity. Finally, we give some conclusions and an outlook in Section 6.

\section{The Reynolds-averaged Navier-Stokes and $k-\omega$ turbulence equations}

We consider the steady-state Reynolds-averaged Navier-Stokes equations and the Wilcox $k-\omega$ turbulence model equations [31, 32],

$$
\nabla \cdot\left(\mathcal{F}^{c}(\mathbf{u})-\mathcal{F}^{v}(\mathbf{u}, \nabla \mathbf{u})\right)-\mathcal{S}(\mathbf{u}, \nabla \mathbf{u})=0 \quad \text { in } \Omega,
$$

on the domain $\Omega \subset \mathbb{R}^{d}, d=2,3$. Similar to $[1,2]$ the equations are considered in terms of the auxiliary variable $\tilde{\omega}=$ $\ln \omega$ instead of $\omega$ for a more moderate near-wall behaviour of the variable. Additionally, this variable transformation guarantees positivity of $\omega$. Then, the vector of conservative variables $\mathbf{u} \in \mathbb{R}^{d+4}$ and the convective fluxes $\mathcal{F}^{c}=$ $\left(\mathbf{f}_{1}^{c}, \ldots, \mathbf{f}_{d}^{c}\right)$ are given by

$$
\mathbf{u}=\left[\begin{array}{c}
\rho \\
\rho v_{i} \\
\rho E \\
\rho k \\
\rho \tilde{w}
\end{array}\right], \quad \mathbf{f}_{j}^{c}(\mathbf{u})=\left[\begin{array}{c}
\rho v_{j} \\
\rho v_{i} v_{j}+\left(p+\frac{2}{3} \rho k\right) \delta_{i j} \\
\left(\rho H-\frac{1}{3} \rho k\right) v_{j} \\
\rho k v_{j} \\
\rho \tilde{w} v_{j}
\end{array}\right], \quad j=1, \ldots, d
$$

where $\rho, \mathbf{v}=\left(v_{1}, \ldots, v_{d}\right)^{\top}, p$ and $E$ denote the density, velocity vector, pressure and specific total energy, respectively. Additionally, $H$ is the total enthalpy given by $H=E+\frac{p}{\rho}$, and the pressure is determined by the equation of state of an ideal gas,

$$
p=(\gamma-1) \rho\left(e_{0}-\frac{1}{2} \mathbf{v}^{2}\right)
$$

with $e_{0}=E-k$. Here, $\gamma=c_{p} / c_{v}$ is the ratio of specific heat capacities at constant pressure, $c_{p}$, and constant volume, $c_{v}$; for dry air $\gamma=1.4$. Furthermore, the viscous fluxes are given by

$$
\mathbf{f}_{j}^{v}(\mathbf{u}, \nabla \mathbf{u})=\left[\begin{array}{c}
0 \\
\bar{\tau}_{i j} \\
\bar{\tau}_{j i} v_{i}+\mathcal{K} T_{x_{j}} \\
\left(\mu+\sigma_{k} \mu_{t}\right) k_{x_{j}} \\
\left(\mu+\sigma_{\omega} \mu_{t}\right) \tilde{\omega}_{x_{j}}
\end{array}\right]
$$

where the thermal conductivity coefficient $\mathcal{K}$ is given by $\mathcal{K}=c_{p}\left(\frac{\mu}{\operatorname{Pr}_{1}}+\frac{\mu_{t}}{\operatorname{Pr}_{t}}\right)$ and the temperature $T$ by $c_{v} T=e=$ $E-\frac{1}{2} \mathbf{v}^{2}-k$. Here $\operatorname{Pr}=0.72$ and $\operatorname{Pr}_{t}=0.9$ are the molecular and turbulent Prandtl numbers, and $\mu$ and $\mu_{t}=C_{\mu} \frac{\rho k}{\omega}$ with $C_{\mu}=1$ are the molecular and turbulent viscosities.

The stress tensor $\underline{\bar{\tau}}$ is given by following relation

$$
\underline{\tau}+\underline{\tau}^{R}=\underline{\bar{\tau}}-\frac{2}{3} \rho k \underline{I}=\underline{\tau}+\underline{\tau}_{t}-\frac{2}{3} \rho k \underline{I}=\left(\mu+\mu_{t}\right) \underline{S}-\frac{2}{3} \rho k \underline{I},
$$


where $\underline{\tau}=\mu \underline{S}$ is the viscous stress tensor, and $\underline{\tau}^{R}=\mu_{t} \underline{S}-\frac{2}{3} \rho k \underline{I}$ is the Reynolds stress tensor with $\underline{S}=\nabla \mathbf{v}+(\nabla \mathbf{v})^{\top}-$ $\frac{2}{3}(\nabla \cdot \mathbf{v}) \underline{I}$. We note, that the viscous flux can be rewritten as $\mathbf{f}_{k}^{v}(\mathbf{u}, \nabla \mathbf{u})=G_{k l}(\mathbf{u}) \partial \mathbf{u} / \partial x_{l}, k=1,2,3$, where the matrices $G_{k l}(\mathbf{u})=\partial \mathbf{f}_{k}^{v}(\mathbf{u}, \nabla \mathbf{u}) / \partial u_{x_{l}}$, for $k, l=1,2,3$, are the homogeneity tensors defined by $\left(\mathbf{f}_{k}^{v}\right)_{i}(\mathbf{u}, \nabla \mathbf{u})=\left(G_{k l}(\mathbf{u})\right)_{i j} \partial u_{j} / \partial x_{l}$, $k=1,2,3, i=1, \ldots, d+4$. Finally, the source term $\mathcal{S}(\mathbf{u}, \nabla \mathbf{u})$ is given by

$$
\mathcal{S}(\mathbf{u}, \nabla \mathbf{u})=\left[\begin{array}{c}
0 \\
\mathbf{0} \\
-\tau_{i j}^{R} v_{i, x_{j}}+\beta_{k} \rho k e^{\tilde{\omega}_{r}} \\
\tau_{i j}^{R} v_{i, x_{j}}-\beta_{k} \rho k e^{\tilde{\omega}_{r}} \\
\alpha_{\omega} \frac{\omega}{k} \tau_{i j}^{R} v_{i, x_{j}}-\beta_{\omega} \rho e^{\tilde{\omega}_{r}}+\left(\mu+\sigma_{\omega} \mu_{t}\right) \tilde{\omega}_{x_{k}} \tilde{\omega}_{x_{k}}
\end{array}\right],
$$

with $\mu_{t}=C_{\mu} \rho k e^{-\tilde{\omega}_{r}}$ and $\tilde{\omega}_{r}=\max \left\{\tilde{\omega}, \tilde{\omega}_{r 0}\right\}$, where $\tilde{\omega}_{r 0}$ fulfills following realizability conditions for the turbulent stresses,

$$
\begin{aligned}
& e^{\tilde{\omega}_{r 0}}-\frac{3}{2} C_{\mu} S_{i i} \geq 0, i=1, \ldots, d, \\
&\left(e^{\tilde{\omega}_{r 0}}\right)^{2}-\frac{3}{2} C_{\mu}\left(S_{i i}+S_{j j}\right) e^{\tilde{\omega}_{r 0}}+\frac{9}{4} C_{\mu}^{2}\left(S_{i i} S_{j j}-S_{i j}^{2}\right) \geq 0, \quad i, j=1, \ldots, d, i \neq j,
\end{aligned}
$$

similar to the realizability conditions given in $[1,2]$. We note that the use of the logarithm of turbulence variables has been introduced in [33] and results in an equivalent reformulation of the Wilcox $k$ - $\omega$ equations $[31,32]$. In the context of DG discretizations it has first been used in [34]. Furthermore, $k$ in the source and destruction terms as well as in the expression for $\mu_{t}$ is kept non-negative. We note, that the limitations on $k$ and $\tilde{\omega}$ avoid unphysical values and have been found in $[1,2]$ to have an stabilizing effect on the numerical scheme. Finally, the values of the $k$ - $\omega$ closure parameters $\alpha_{\omega}=\frac{5}{9}, \beta_{k}=\frac{9}{100}, \beta_{\omega}=\frac{3}{40}, \sigma_{k}=\sigma_{\omega}=\frac{1}{2}$ are those of the high-Reynolds Wilcox $k$ - $\omega$ model $[31,32]$.

\section{The discontinuous Galerkin discretization}

In this section we describe the discontinuous Galerkin discretization used to discretize the RANS- $k \omega$ equations (1). In particular, we employ the second DG scheme proposed by Bassi \& Rebay [35, 36], modified according to the adjoint-consistent treatment of boundary terms and force coefficients as given in $[38,37]$.

We assume that $\Omega$ can be subdivided into shape-regular meshes $\mathcal{T}_{h}=\{k\}$ consisting of (possibly curved) quadrilateral or hexahedral elements $\kappa$. Furthermore, we assume that each $\kappa \in \mathcal{T}_{h}$ is an image of a fixed reference element $\hat{\kappa}$, that is, $\kappa=\sigma_{\kappa}(\hat{\kappa})$ for all $\kappa \in \mathcal{T}_{h}$, where $\hat{\kappa}$ is the open unit square in $\mathbb{R}^{2}$ and the open unit cube in $\mathbb{R}^{3}$ and $\sigma_{\kappa}$ is a smooth bijective mapping. In order to allow boundary elements to be curved the mapping $\sigma_{\kappa}$ is constructed based on employing a higher degree polynomial representation of the computational boundary (e. g., see $[14,39,40])$. Furthermore, we allow interior elements to be curved in order to avoid the intersection of curved boundary lines with interior elements [7], which might occur for meshes with highly stretched elements as typically used for turbulent flows.

On the reference element $\hat{\kappa}$ we define the space of polynomials of degree $p \geq 0$ as follows:

$$
\mathcal{P}_{p}=\operatorname{span}\left\{\hat{\mathbf{x}}^{\alpha}: 0 \leq|\alpha| \leq p\right\} .
$$

We now introduce the finite element space $\mathbf{V}_{h, p}$ consisting of discontinuous vector-valued piecewise polynomial functions of degree $p \geq 0$, as follows:

$$
\mathbf{V}_{h, p}=\left\{\mathbf{v}_{h} \in\left[L_{2}(\Omega)\right]^{d+4}:\left.\mathbf{v}_{h}\right|_{\kappa} \circ \sigma_{\kappa} \in\left[\mathcal{P}_{p}(\hat{\kappa})\right]^{d+4}, \kappa \in \mathcal{T}_{h}\right\} .
$$

An interior face of $\mathcal{T}_{h}$ is defined as the (non-empty) two-dimensional interior of $\partial \kappa^{+} \cap \partial \kappa^{-}$, where $\kappa^{+}$and $\kappa^{-}$are two adjacent elements of $\mathcal{T}_{h}$. A boundary face of $\mathcal{T}_{h}$ is defined as the (non-empty) two-dimensional interior of $\partial \kappa \cap \Gamma$, where $\kappa$ is a boundary element of $\mathcal{T}_{h}$. We denote by $\Gamma_{I}$ the union of all interior faces of $\mathcal{T}_{h}$. Furthermore, we define some jump and mean value operators for vector- and matrix-valued functions. To this end, let $\kappa^{+}$and $\kappa^{-}$be two adjacent elements of $\mathcal{T}_{h}$ and $\mathbf{x}$ be an arbitrary point on the interior face $f=\partial \kappa^{+} \cap \partial \kappa^{-} \subset \Gamma_{\mathcal{I}}$. Moreover, let $\mathbf{v}$ and $\underline{\tau}$ be vector- and matrix-valued functions, respectively, that are smooth inside each element $\kappa^{ \pm}$. By $\mathbf{v}^{ \pm}:=\left.\mathbf{v}\right|_{\partial \kappa^{ \pm}}$and $\bar{\tau}^{ \pm}:=\left.\underline{\tau}\right|_{\partial k^{ \pm}}$we denote the traces of, respectively, $\mathbf{v}$ and $\underline{\tau}$ on $f$ taken from within the interior of $\kappa^{ \pm}$, respectively. 
Then, we define the averages at $\mathbf{x} \in f$ by $\{\mathbf{v}\}=\left(\mathbf{v}^{+}+\mathbf{v}^{-}\right) / 2$ and $\{\underline{\tau}\}=\left(\underline{\tau}^{+}+\underline{\tau}^{-}\right) / 2$. Similarly, the jump at $\mathbf{x} \in f$ is given by $\underline{\llbracket \mathbf{v} \rrbracket}=\mathbf{v}^{+} \otimes \mathbf{n}_{\kappa^{+}}+\mathbf{v}^{-} \otimes \mathbf{n}_{K^{-}}$. On a boundary face $f \subset \Gamma$, we set $\{\mathbf{v}\}=\mathbf{v},\{\underline{\tau}\}=\underline{\tau}$ and $\llbracket \mathbf{v} \rrbracket=\mathbf{v} \otimes \mathbf{n}$. For matrices $\underline{\sigma}, \underline{\tau} \in \mathbb{R}^{m \times n}, m, n \geq 1$, we use the standard notation $\underline{\sigma}: \underline{\tau}=\sum_{k=1}^{m} \sum_{l=1}^{n} \sigma_{k l} \tau_{k l}$; additionally, for vectors $\mathbf{v} \in \mathbb{R}^{m}, \mathbf{w} \in \mathbb{R}^{n}$, the matrix $\mathbf{v} \otimes \mathbf{w} \in \mathbb{R}^{m \times n}$ is defined by $(\mathbf{v} \otimes \mathbf{w})_{k l}=v_{k} w_{l}$. Furthermore, we use the notation $G^{\top}(\mathbf{u}) \nabla \mathbf{v}: \mathbf{u} \otimes \mathbf{n}=\left(G_{k l}(\mathbf{u})\right)_{i j} \partial v_{i} / \partial x_{k} u_{j} n_{l}$.

Then the discontinuous Galerkin discretization of (1) is given by: Find $\mathbf{u}_{h} \in \mathbf{V}_{h, p}$ such that

$$
\begin{aligned}
\mathcal{N}\left(\mathbf{u}_{h}, \mathbf{v}\right) \equiv \int_{\Omega}\left(-\mathcal{F}^{c}\left(\mathbf{u}_{h}\right)+\mathcal{F}^{v}\left(\mathbf{u}_{h}, \nabla_{h} \mathbf{u}_{h}\right)\right): \nabla_{h} \mathbf{v}-\mathcal{S}\left(\mathbf{u}_{h}, \nabla_{h} \mathbf{u}_{h}\right) \cdot \mathbf{v} \mathrm{d} \mathbf{x}+\sum_{\kappa \in \mathcal{T}_{h}} \int_{\partial \kappa \backslash \Gamma} \mathcal{H}\left(\mathbf{u}_{h}^{+}, \mathbf{u}_{h}^{-}, \mathbf{n}^{+}\right) \cdot \mathbf{v}^{+} \mathrm{d} s \\
\quad-\int_{\Gamma_{I}}\left\{\mathcal{F}^{v}\left(\mathbf{u}_{h}, \nabla_{h} \mathbf{u}_{h}\right)\right\}: \underline{\llbracket \mathbf{v} \rrbracket} \mathrm{d} s-\int_{\Gamma_{I}}\left\{G^{\top}\left(\mathbf{u}_{h}\right) \nabla_{h} \mathbf{v}\right\}: \underline{\llbracket \mathbf{u}_{h} \rrbracket} \mathrm{d} s+\int_{\Gamma_{I}} \underline{\delta}\left(\mathbf{u}_{h}\right): \underline{\llbracket \mathbf{v} \rrbracket} \mathrm{d} s+\mathcal{N}_{\Gamma}\left(\mathbf{u}_{h}, \mathbf{v}\right)=0
\end{aligned}
$$

for all $\mathbf{v}$ in $\mathbf{V}_{h, p}$. Here, $\mathcal{H}$ denotes any consistent, conservative and two-point monotone numerical flux function; we note that in Section 5 the local Lax-Friedrichs flux is employed. For the penalization term we consider the second scheme of Bassi and Rebay (BR2), [35, 36]:

$$
\underline{\delta}\left(\mathbf{u}_{h}\right)=\underline{\delta}^{\mathrm{BR} 2}\left(\mathbf{u}_{h}\right)=C_{\mathrm{BR} 2}\left\{\underline{L}_{0}^{f}\left(\mathbf{u}_{h}\right)\right\},
$$

where the local lifting operator $\underline{L}_{0}^{f}\left(\mathbf{u}_{h}\right) \in \underline{\Sigma}_{h, p}$ is defined by:

$$
\int_{\Omega_{f}} \underline{L}_{0}^{f}\left(\mathbf{u}_{h}\right): \underline{\tau} \mathrm{d} \mathbf{x}=\int_{f} \underline{\llbracket \mathbf{u}_{h} \rrbracket}:\left\{G^{\top}\left(\mathbf{u}_{h}\right) \underline{\tau}\right\} \mathrm{d} s \quad \forall \underline{\tau} \in \underline{\Sigma}_{h, p},
$$

where $\Omega_{f}=\kappa_{f}^{+} \cup \kappa_{f}^{-}$for the interior face $f=\partial \kappa_{f}^{+} \cap \partial \kappa_{f}^{-} \in \Gamma_{I}$.

Finally, the boundary terms included in $\mathcal{N}_{\Gamma}\left(\mathbf{u}_{h}, \mathbf{v}\right)$ are given by

$$
\begin{aligned}
\mathcal{N}_{\Gamma}\left(\mathbf{u}_{h}, \mathbf{v}\right)= & \int_{\Gamma} \mathcal{H}_{\Gamma}\left(\mathbf{u}_{h}^{+}, \mathbf{u}_{\Gamma}\left(\mathbf{u}_{h}^{+}\right), \mathbf{n}^{+}\right) \cdot \mathbf{v}^{+} \mathrm{d} s+\int_{\Gamma} \underline{\delta}_{\Gamma}\left(\mathbf{u}_{h}^{+}\right): \mathbf{v} \otimes \mathbf{n} \mathrm{d} s \\
& -\int_{\Gamma} \mathbf{n} \cdot \mathcal{F}_{\Gamma}^{v}\left(\mathbf{u}_{h}^{+}, \nabla_{h} \mathbf{u}_{h}^{+}\right) \mathbf{v}^{+} \mathrm{d} s-\int_{\Gamma}\left(G_{\Gamma}^{\top}\left(\mathbf{u}_{h}^{+}\right) \nabla_{h} \mathbf{v}_{h}^{+}\right):\left(\mathbf{u}_{h}^{+}-\mathbf{u}_{\Gamma}\left(\mathbf{u}_{h}^{+}\right)\right) \otimes \mathbf{n} \mathrm{d} s .
\end{aligned}
$$

The penalization term on the boundary is given by $\underline{\delta}_{\Gamma}\left(\mathbf{u}_{h}\right)=C_{\mathrm{BR} 2} \underline{L}_{\Gamma}^{f}\left(\mathbf{u}_{h}\right)$ where the local lifting operator $\underline{L}_{\Gamma}^{f}\left(\mathbf{u}_{h}\right) \in \underline{\Sigma}_{h, p}$ is defined by:

$$
\int_{\kappa} \underline{L}_{\Gamma}^{f}\left(\mathbf{u}_{h}\right): \underline{\tau} \mathrm{d} \mathbf{x}=\int_{f}\left(\mathbf{u}_{h}-\mathbf{u}_{\Gamma}\left(\mathbf{u}_{h}\right)\right) \otimes \mathbf{n}:\left(G_{\Gamma}^{\top}\left(\mathbf{u}_{h}\right) \underline{\tau}\right) \mathrm{d} s \quad \forall \underline{\tau} \in \underline{\Sigma}_{h, p}
$$

for all $\kappa \in \mathcal{T}_{h}$, such that $\partial \kappa \cap \Gamma=f$. Furthermore, the viscous boundary flux $\mathcal{F}_{\Gamma}^{v}$ and the corresponding homogeneity tensor $G_{\Gamma}$ are given by

$$
\mathcal{F}_{\Gamma}^{v}\left(\mathbf{u}_{h}, \nabla \mathbf{u}_{h}\right)=\mathcal{F}^{v}\left(\mathbf{u}_{\Gamma}\left(\mathbf{u}_{h}\right), \nabla \mathbf{u}_{h}\right)=G_{\Gamma}\left(\mathbf{u}_{h}\right) \nabla \mathbf{u}_{h}=G\left(\mathbf{u}_{\Gamma}\left(\mathbf{u}_{h}\right)\right) \nabla \mathbf{u}_{h} .
$$

Furthermore, on adiabatic boundaries $\mathcal{F}_{\Gamma}^{v}$ and $G_{\Gamma}$ are modified such that $\mathbf{n} \cdot \nabla T=0$. Finally, the numerical flux $\mathcal{H}_{\Gamma}$ at the boundary is given by

$$
\mathcal{H}_{\Gamma}\left(\mathbf{u}_{h}^{+}, \mathbf{u}_{\Gamma}\left(\mathbf{u}_{h}^{+}\right), \mathbf{n}\right)=\mathbf{n} \cdot \mathcal{F}_{\Gamma}^{c}\left(\mathbf{u}_{h}^{+}\right)=\mathbf{n} \cdot \mathcal{F}^{c}\left(\mathbf{u}_{\Gamma}\left(\mathbf{u}_{h}^{+}\right)\right),
$$

where the boundary function is $\mathbf{u}_{\Gamma}(\mathbf{u})=\left(u_{1}, 0,0,0, u_{5}, 0, u_{1} \omega_{\text {wall }}\right)^{\top}$. Here, the value of $\omega_{\text {wall }}$ is determined by Menter's boundary condition (see [41]), with $\omega_{\text {wall }}=\frac{10 \cdot 6 v}{\beta y_{1}^{2}}$, where $v=\frac{\mu}{\rho}$ is the kinematic viscosity, and $y_{1}$ denotes the value of the first wall boundary layer grid spacing.

\section{Error estimation and adjoint-based refinement}

In this section we recall the adjoint-based error estimation and adaptive mesh refinement approach for single target quantities (e. g., see [13, 15]) and for the treatment of multiple target quantities (see [27, 28]). 
Given a target quantity $J(\mathbf{u})$ such as the aerodynamic drag, lift or moment coefficient, a duality argument can be employed, to obtain following error representation, $[13,15]$,

$$
J(\mathbf{u})-J\left(\mathbf{u}_{h}\right)=-\mathcal{N}\left(\mathbf{u}_{h}, \mathbf{z}\right) \equiv \mathcal{R}\left(\mathbf{u}_{h}, \mathbf{z}\right) \approx \mathcal{R}\left(\mathbf{u}_{h}, \tilde{\mathbf{z}}_{h}\right)
$$

where the exact adjoint solution $\mathbf{z}$ is replaced by the solution $\tilde{\mathbf{z}}_{h}$ to the following discrete adjoint problem: Find $\tilde{\mathbf{z}}_{h} \in \tilde{\mathbf{V}}_{h, p}$ such that

$$
\mathcal{N}^{\prime}\left[\mathbf{u}_{h}\right]\left(\mathbf{w}_{h}, \tilde{\mathbf{z}}_{h}\right)=J^{\prime}\left[\mathbf{u}_{h}\right]\left(\mathbf{w}_{h}\right) \quad \forall \mathbf{w}_{h} \in \tilde{\mathbf{V}}_{h, p} .
$$

A possible choice of the adjoint discrete function space is $\tilde{\mathbf{V}}_{h, p}=\mathbf{V}_{h, p+1}$ (see also the Remark below). The approximate error representation in (7) can then be localized

$$
J(\mathbf{u})-J\left(\mathbf{u}_{h}\right) \approx \mathcal{R}\left(\mathbf{u}_{h}, \tilde{\mathbf{z}}_{h}\right) \equiv \sum_{\kappa \in \mathcal{T}_{h}} \tilde{\eta}_{\kappa},
$$

where $\tilde{\eta}_{\kappa}$ are the so-called adjoint-based indicators which include the local residuals multiplied by the discrete adjoint solution. These indicators can be used to drive an adaptive mesh refinement algorithm tailored to the accurate and efficient approximation of the target quantity $J(\mathbf{u})$ under consideration. Finally, the approximate error representation (9) can be used to enhance the computed target quantity $J\left(\mathbf{u}_{h}\right)$ as follows:

$$
\tilde{J}\left(\mathbf{u}_{h}\right)=J\left(\mathbf{u}_{h}\right)+\mathcal{R}\left(\mathbf{u}_{h}, \tilde{\mathbf{z}}_{h}\right) .
$$

Remark 1. We note that in Galerkin finite element methods the Galerkin orthogonality can be used to subtract any discrete function $\mathbf{z}_{h} \in \mathbf{V}_{h, p}$ from the adjoint solution in the error estimate (7) resulting in $J(\mathbf{u})-J\left(\mathbf{u}_{h}\right)=-\mathcal{N}\left(\mathbf{u}_{h}, \mathbf{z}-\mathbf{z}_{h}\right)$ (e.g., see [13]). In order to avoid a vanishing error estimate (7), the discrete adjoint solution $\tilde{\mathbf{z}}_{h} \in \tilde{\mathbf{V}}_{h, p}$ must be computed in a richer space (e.g., $\tilde{\mathbf{V}}_{h, p}=\mathbf{V}_{h, p+1}$ ) than the flow solution $\mathbf{u}_{h} \in \mathbf{V}_{h, p}$. In continuous finite element methods it is required to choose $\mathbf{z}_{h}$ to be an approximation of $\mathbf{z}$ in order to ensure that the local indicators in (9) are of the right order of convergence [13]. For continuous finite element methods Galerkin orthogonality is a global property (i.e., it holds true on the whole domain). In addition to that discontinuous Galerkin methods satisfy a Galerkin orthogonality on a local, element-wise level. Therefore, for DG methods $\mathbf{z}_{h}$ can be omitted without changing the local indicators.

The extension of the adjoint-based error estimation and mesh refinement approach to multiple target quantities has previously been considered for the inviscid Burgers' equation in [27] and has been extended to two-dimensional viscous laminar compressible flows in [28]. Estimating the error in multiple quantities of interest, $J_{i}(\mathbf{u}), i=1, \ldots, N$, would require the computation of the solutions $\tilde{\mathbf{z}}_{h, i} \in \tilde{\mathbf{V}}_{h, p}$ to $N$ discrete adjoint problems:

$$
\mathcal{N}^{\prime}\left[\mathbf{u}_{h}\right]\left(\mathbf{w}_{h}, \tilde{\mathbf{z}}_{h, i}\right)=J_{i}^{\prime}\left[\mathbf{u}_{h}\right]\left(\mathbf{w}_{h}\right) \quad \forall \mathbf{w}_{h} \in \tilde{\mathbf{V}}_{h, p}, \quad i=1, \ldots, N,
$$

and the evaluation of the error representation for each of the quantities,

$$
J(\mathbf{u})-J\left(\mathbf{u}_{h}\right) \approx \mathcal{R}\left(\mathbf{u}_{h}, \tilde{\mathbf{z}}_{h, i}\right), \quad i=1, \ldots, N .
$$

Instead, we compute the solution to the following discrete error equation: Find $\tilde{\mathbf{e}}_{h} \in \tilde{\mathbf{V}}_{h, p}$ such that

$$
\mathcal{N}^{\prime}\left[\mathbf{u}_{h}\right]\left(\tilde{\mathbf{e}}_{h}, \mathbf{w}_{h}\right)=\mathcal{R}\left(\mathbf{u}_{h}, \mathbf{w}_{h}\right) \quad \forall \mathbf{w}_{h} \in \tilde{\mathbf{V}}_{h, p},
$$

and evaluate the following approximation of $J_{i}(\mathbf{u})-J_{i}\left(\mathbf{u}_{h}\right)$,

$$
J_{i}(\mathbf{u})-J_{i}\left(\mathbf{u}_{h}\right) \approx J_{i}^{\prime}\left[\mathbf{u}_{h}\right](\mathbf{e}) \approx J_{i}^{\prime}\left[\mathbf{u}_{h}\right]\left(\tilde{\mathbf{e}}_{h}\right), \quad i=1, \ldots, N,
$$

where $\mathbf{e}=\mathbf{u}-\mathbf{u}_{h}$. Furthermore, based on a suitable combination $J_{c}(\mathbf{u})$ of the original target quantities, we compute the solution to the following discrete adjoint problem: Find $\tilde{\mathbf{z}}_{c, h} \in \tilde{\mathbf{V}}_{h, p}$ such that

$$
\mathcal{N}^{\prime}\left[\mathbf{u}_{h}\right]\left(\mathbf{w}_{h}, \tilde{\mathbf{z}}_{c, h}\right)=J_{c}^{\prime}\left[\mathbf{u}_{h}\right]\left(\mathbf{w}_{h}\right) \quad \forall \mathbf{w}_{h} \in \tilde{\mathbf{V}}_{h, p}
$$


and evaluate the error estimate

$$
J_{c}(\mathbf{u})-J_{c}\left(\mathbf{u}_{h}\right)=\mathcal{R}\left(\mathbf{u}_{h}, \mathbf{z}_{c}\right) \approx \mathcal{R}\left(\mathbf{u}_{h}, \tilde{\mathbf{z}}_{c, h}\right) \equiv \sum_{\kappa \in \mathcal{T}_{h}} \tilde{\eta}_{\kappa}^{c}
$$

The combined target quantity $J_{c}(\mathbf{u})$ can be defined [28] such that the error with respect to $J_{c}(\cdot)$ represents the sum of relative errors in the original target quantities, $\sum_{i=1}^{N}\left|J_{i}(\mathbf{u})-J_{i}\left(\mathbf{u}_{h}\right)\right| /\left|J_{i}\left(\mathbf{u}_{h}\right)\right|$, or a weighted sum of absolute errors

$$
\sum_{i=1}^{N} \alpha_{i}\left|J_{i}(\mathbf{u})-J_{i}\left(\mathbf{u}_{h}\right)\right|
$$

with weighting factors $\alpha_{i}>0$. The adjoint-based indicators, $\tilde{\eta}_{\kappa}^{c}$, obtained by localizing the estimate (14), can be used to drive an adaptive algorithm for the accurate and efficient approximation of all the target quantities, $J_{i}(\mathbf{u}), i=1, \ldots, N$, under consideration.

Finally, we note that the error estimates of (12) can be used to enhance the computed target quantities $J_{i}\left(\mathbf{u}_{h}\right)$, $i=1, \ldots, N$, as follows:

$$
\tilde{J}_{i}\left(\mathbf{u}_{h}\right)=J_{i}\left(\mathbf{u}_{h}\right)+J_{i}^{\prime}\left[\mathbf{u}_{h}\right]\left(\tilde{\mathbf{e}}_{h}\right), \quad i=1, \ldots, N
$$

\section{Numerical results}

In this section we consider the application of the adjoint-based error estimation and goal-oriented mesh refinement approach described in Section 4 to three aerodynamic test cases of increasing complexity which have been considered in the EU project ADIGMA [11]. In particular, we consider turbulent flows around a three-dimensional streamlined body, the three-element L1T2 high-lift configuration and the DLR-F6 wing-body configuration. In the following, adjoint-based mesh refinement is always combined with anisotropic mesh refinement. On elements which are flagged for refinement by using the adjoint-based indicators $\tilde{\eta}_{\kappa}, \kappa \in \mathcal{T}_{h}$, (see Equations (9) or (14)) an anisotropic jump indicator is employed to decide along which direction the element shall be refined. In particular, a quadrilateral or hexahedral element is refined along a specific direction if the average jump of the discrete function $\mathbf{u}_{h} \in \mathbf{V}_{h, p}$ over the two opposite faces in that direction is not significantly smaller than the average jumps over the remaining pairs of opposite faces. As a result an element is refined isotropically if the average jumps are of similar size; otherwise the element is refined anisotropically. This anisotropic refinement strategy has previously been developed for $2 \mathrm{~d}$ and $3 \mathrm{~d}$ laminar flows in $[21,22]$. Including all flow variables in the average jumps it can also be applied to the $2 \mathrm{~d}$ and $3 \mathrm{~d}$ turbulent flows considered in the following.

\subsection{Turbulent flow around a streamlined body}

We begin by considering a turbulent flow around a streamlined three-dimensional body based on a 10 percent thick airfoil with boundaries constructed by a surface of revolution. In particular, we consider the streamlined body at a Mach number $M=0.5$, an angle of attack $\alpha=5^{\circ}$, and a Reynolds number $\operatorname{Re}=10 \cdot 10^{6}$ with adiabatic noslip wall boundary conditions. This is the ADIGMA BTC0 test case which has been defined in the ADIGMA project [11] in order to enable grid convergence studies. Based on extrapolation of higher order computational results and of the results on very fine locally adapted meshes, following reference values of the total drag, lift, and pitching moment coefficients, $C_{\mathrm{d}}, C_{1}$ and $C_{\text {my }}$ have been obtained: $J_{C_{1}}(\mathbf{u})=0.00663, J_{C_{\mathrm{d}}}(\mathbf{u})=0.00858$ and $J_{C_{\mathrm{my}}}(\mathbf{u})=0.00588$.

In the following the total drag, lift, and pitching moment coefficients will be computed up to a predefined error tolerance TOL. Let us consider the following accuracy requirements:

$$
\begin{aligned}
& \left|J_{C_{1}}(\mathbf{u})-J_{C_{1}}\left(\mathbf{u}_{h}\right)\right| \leq \mathrm{TOL}_{C_{1}}=3 \cdot 10^{-4}, \\
& \left|J_{C_{\mathrm{d}}}(\mathbf{u})-J_{C_{\mathrm{d}}}\left(\mathbf{u}_{h}\right)\right| \leq \mathrm{TOL}_{C_{\mathrm{d}}}=1 \cdot 10^{-4} \text {, } \\
& \left|J_{C_{\mathrm{my}}}(\mathbf{u})-J_{C_{\mathrm{my}}}\left(\mathbf{u}_{h}\right)\right| \leq \mathrm{TOL}_{C_{\mathrm{my}}}=2 \cdot 10^{-4} \text {. }
\end{aligned}
$$

In the following we compare the performance in meeting these accuracy requirements for higher order discretizations against second order discretizations on globally refined meshes. We also compare against the performance of the two goal-oriented refinement strategies presented in Section 4. In particular, we consider the single-target error 


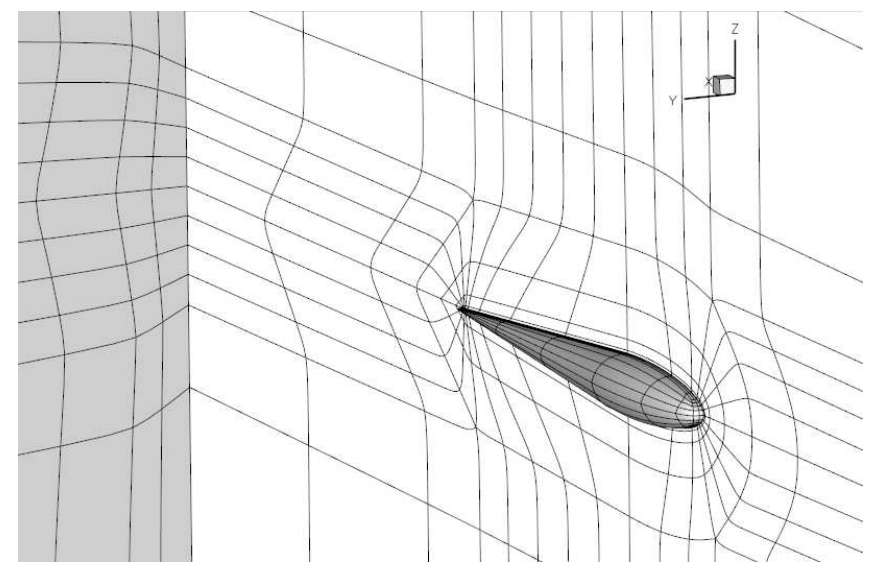

Figure 1: ADIGMA BTC0 test case at turbulent conditions: Body surface, symmetry plane and cut through the domain of the coarse mesh with 6656 curved elements. The lines are given by polynomials of degree 4 .

estimation and mesh refinement approach for each of the $C_{\mathrm{l}}, C_{\mathrm{d}}$, and $C_{\mathrm{my}}$ coefficients, separately. This results in three different sequences of locally refined meshes where on each mesh a flow problem (2) and a discrete adjoint problem (8) are solved, and the error estimate (7) is evaluated. Furthermore, we consider a multi-target error estimation and mesh refinement approach for reducing a weighted sum of absolute errors of the $C_{1}, C_{\mathrm{d}}$ and $C_{\mathrm{my}}$ values. This yields one sequence of locally refined meshes that targets at reducing the error in all three coefficients simultaneously. Here, on each mesh a flow problem (2), a discrete error equation (11) and a discrete adjoint problem (13) are solved, and the error estimates (12) and (14) are evaluated. The weighting factors in the weighted sum (15) might account for the different tolerances in (17). In fact, in the following, the multi-target approach will be based on the weighted sum

$$
2\left|J_{C_{1}}(\mathbf{u})-J_{C_{1}}\left(\mathbf{u}_{h}\right)\right|+6\left|J_{C_{\mathrm{d}}}(\mathbf{u})-J_{C_{\mathrm{d}}}\left(\mathbf{u}_{h}\right)\right|+3\left|J_{C_{\mathrm{my}}}(\mathbf{u})-J_{C_{\mathrm{my}}}\left(\mathbf{u}_{h}\right)\right| .
$$

Figure 1 shows the starting mesh of this computation with 6656 curved elements. The edges are given by polynomials of degree 4 based on additional points taken from finer grids with straight edges. On this mesh we first compute the flow solutions $\mathbf{u}_{h} \in \mathbf{V}_{h, p}$ for the polynomial degrees $p=1, \ldots, 4$. Additionally, for the lower polynomial degrees, we compute the solutions on globally refined meshes. The resulting force coefficients $C_{\mathrm{l}}, C_{\mathrm{d}}$ and $C_{\mathrm{my}}$ plotted over the number of degrees of freedom are given in Figures 2(a), 2(c) and 2(e), respectively. We note that here and throughout this work numbers of degrees of freedom always refer to the total number of degrees of freedom (including all components) of flow solutions $\mathbf{u}_{h} \in \mathbf{V}_{h, p}$. In Figure 2(a) we see that the $p=2,3$ and 4 solutions are within the prescribed accuracy tolerance of the $C_{1}$ value on the coarsest mesh, and they require significantly less degrees of freedom than the $p=1$ solution on the twice globally refined mesh. A similar behaviour is observed in Figure 2(e) for the $C_{\mathrm{m}}$ value. Furthermore, from Figure 2(c) we see that the $p=3$ and 4 solutions on the coarsest mesh are within the prescribed accuracy tolerance of the $C_{\mathrm{d}}$ value.

Let us now consider the adjoint-based error estimation and goal-oriented mesh refinement that targets the lift coefficient $C_{1}$ (i.e., the single target quantity is $J(\mathbf{u})=J_{C_{1}}(\mathbf{u})$ ). To this end we compute the solution $\mathbf{u}_{h} \in \mathbf{V}_{h, 1}$ to the flow problem (2) and the solution $\tilde{\mathbf{z}}_{h} \in \mathbf{V}_{h, 2}$ to the discrete adjoint problem (8). We then evaluate the approximate error representation (9) and obtain the adjoint-based indicators $\tilde{\eta}_{\kappa}$ which we employ for adaptive mesh refinement. Starting on the coarse mesh of 6656 curved elements shown in Figure 1 a sequence of locally refined meshes specifically tailored to the accurate approximation of the $C_{1}$ value is obtained. In Table 1 we collect the number of elements, the (total) number of degrees of freedom (DoF) of $\mathbf{u}_{h} \in \mathbf{V}_{h, 1}$, the true error $J_{C_{1}}(\mathbf{u})-J_{C_{1}}\left(\mathbf{u}_{h}\right)$ in the lift coefficient, the estimated error $\mathcal{R}\left(\mathbf{u}_{h}, \tilde{\mathbf{z}}_{h}\right)$, and the quotient $\theta=\mathcal{R}\left(\mathbf{u}_{h}, \tilde{\mathbf{z}}_{h}\right) /\left(J_{C_{1}}(\mathbf{u})-J_{C_{1}}\left(\mathbf{u}_{h}\right)\right)$ of the estimated and the true error, which is also called the effectivity index. Here, we see that the estimated errors are very close to the true errors. This is also indicated by the effectivity indices which are very close to one. In fact, from the third mesh onwards the rounded index equals one. We note that this represents an almost perfect error estimation. Given the complexity of the governing RANS- $k \omega$ flow equations this might seem surprising. However, the turbulent flow considered here is 


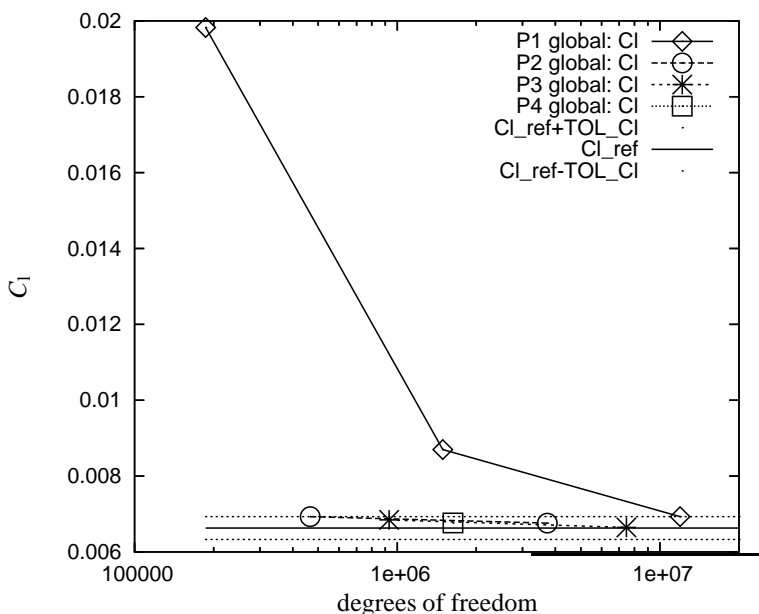

(a)

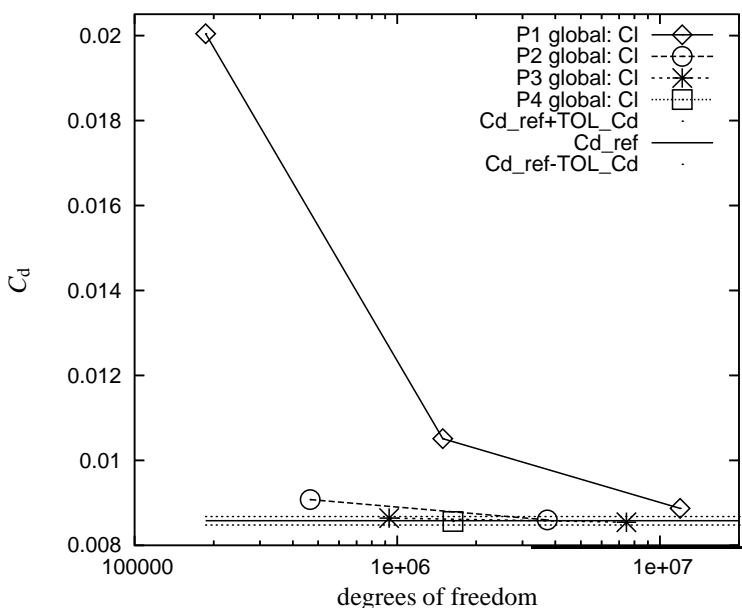

(c)

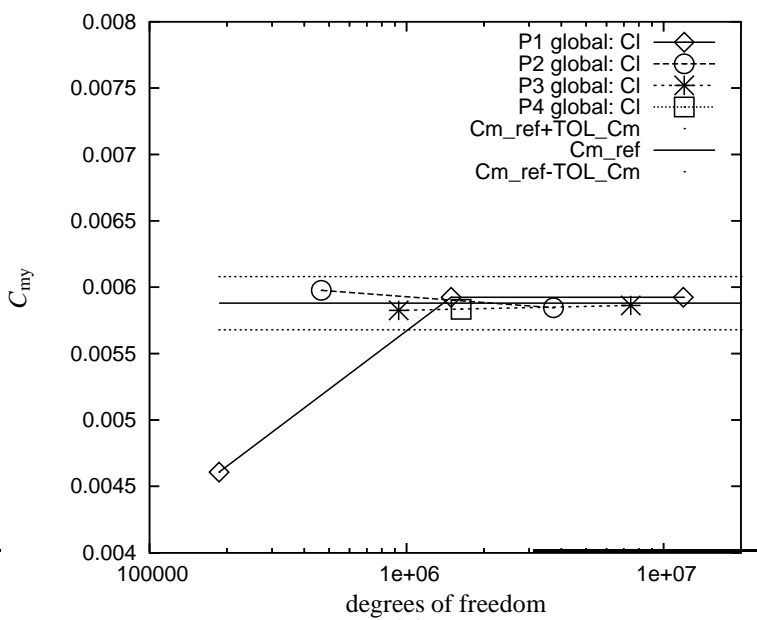

(e)

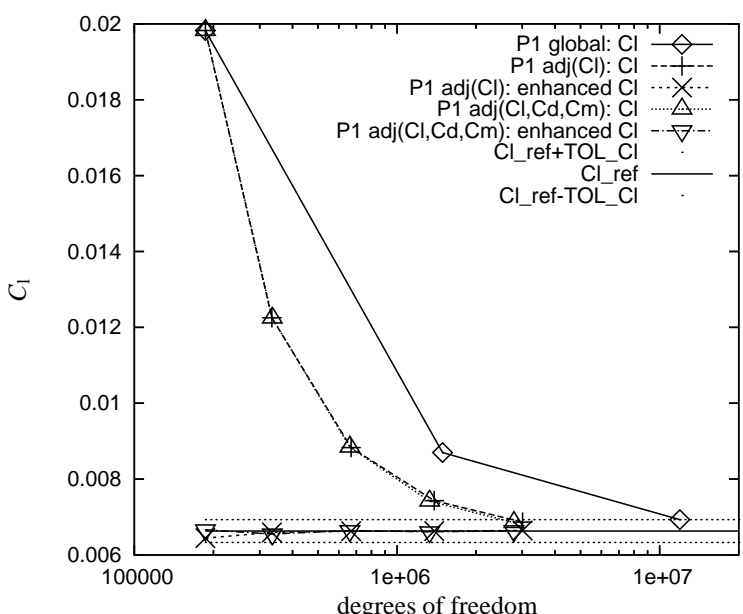

(b)

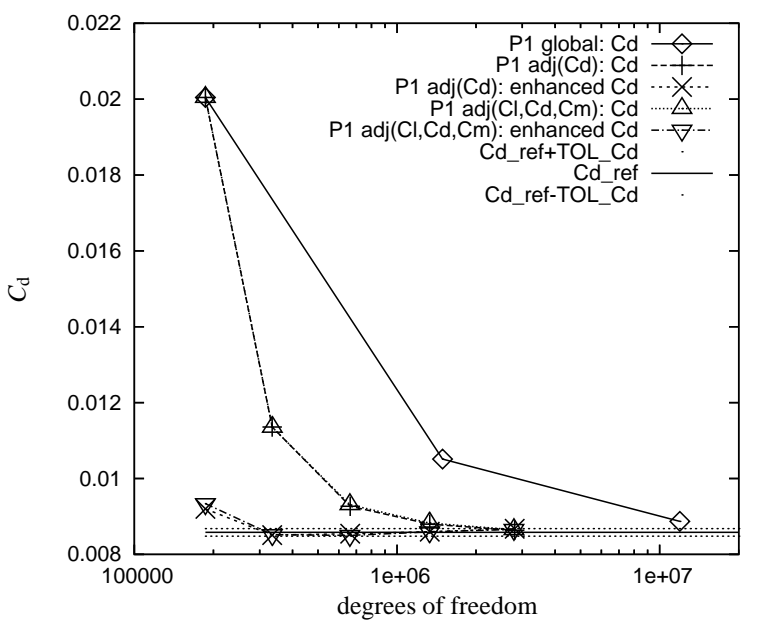

(d)

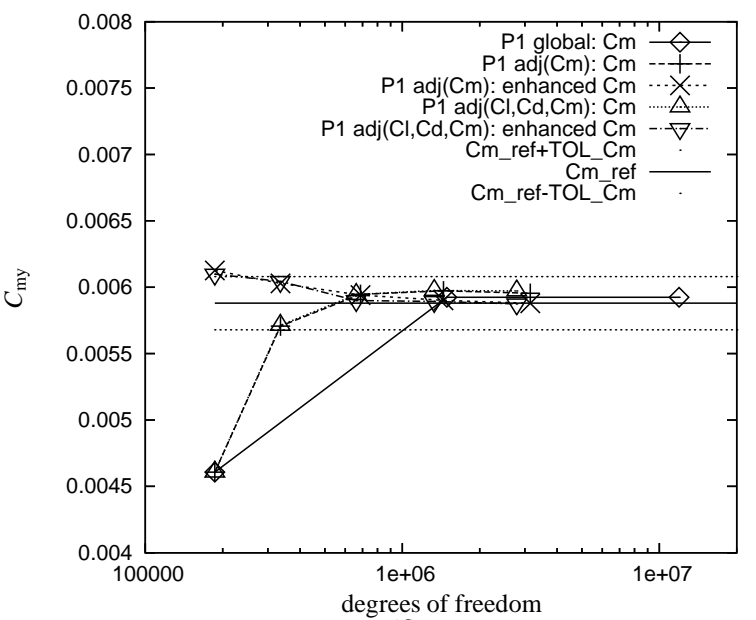

(f)

Figure 2: ADIGMA BTC0 test case at turbulent conditions: $C_{\mathrm{l}}, C_{\mathrm{d}}$ and $C_{\mathrm{my}}$ values in the top, middle and bottom row, respectively. (left) The $p=1,2,3$ solutions on globally refined meshes; (right) The force coefficients $J\left(\mathbf{u}_{h}\right)$ and the enhanced force coefficients, (10) and (16), on the single-target and multi-target adjoint-based refined meshes. 


\begin{tabular}{c|c|c|c|c} 
\# Elements & \# DoF & $J_{C_{1}}(\mathbf{u})-J_{C_{1}}\left(\mathbf{u}_{h}\right)$ & $\mathcal{R}\left(\mathbf{u}_{h}, \tilde{\mathbf{z}}_{h}\right)$ & $\theta$ \\
\hline 6656 & 186368 & $-1.320 \mathrm{e}-02$ & $-1.338 \mathrm{e}-02$ & 1.01 \\
11919 & 333732 & $-5.622 \mathrm{e}-03$ & $-5.661 \mathrm{e}-03$ & 1.01 \\
23894 & 669032 & $-2.200 \mathrm{e}-03$ & $-2.203 \mathrm{e}-03$ & 1.00 \\
49478 & 1385384 & $-8.007 \mathrm{e}-04$ & $-8.011 \mathrm{e}-04$ & 1.00
\end{tabular}

Table 1: ADIGMA BTC0 case at turbulent conditions: Error estimation for the $C_{1}$ value.

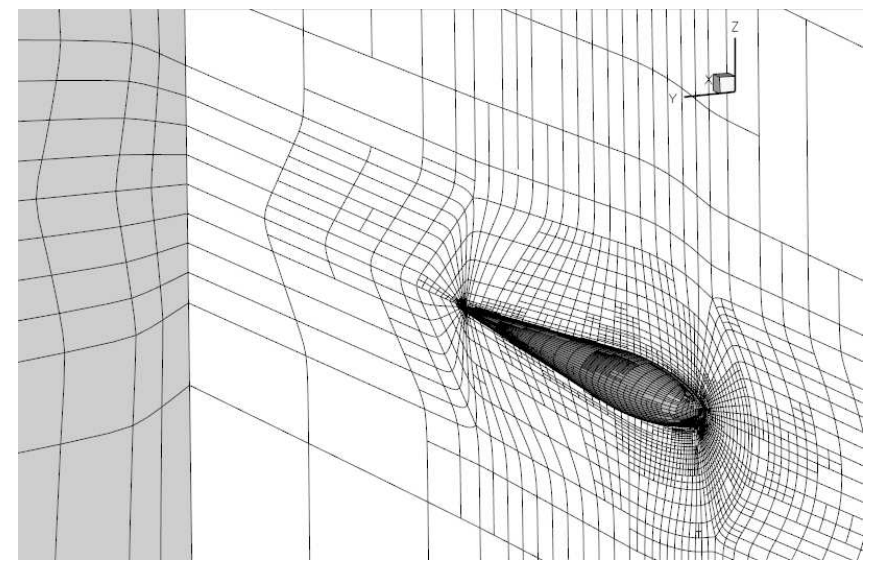

Figure 3: ADIGMA BTC0 test case at turbulent conditions: Mesh with 47497 curved elements after 3 multi-target adjoint-based refinement steps.

particularly smooth. So we expect the error estimation to work very well in this case.

Figure 2(b) shows the $C_{1}$ values on the sequence of adaptively refined meshes. For comparison it also includes the $C_{1}$ values of the $\mathbf{u}_{h} \in \mathbf{V}_{h, 1}$ flow solutions on the globally refined meshes already shown in Figure 2(a). Furthermore, Figure 2(b) includes the enhanced target quantities (10) evaluated based on the computed $C_{1}$ values and the error estimates $\mathcal{R}\left(\mathbf{u}_{h}, \tilde{\mathbf{z}}_{h}\right)$ given in Table 1 . Here, we see that the enhanced quantity $\tilde{J}_{C_{1}}\left(\mathbf{u}_{h}\right)=J_{C_{1}}\left(\mathbf{u}_{h}\right)+\mathcal{R}\left(\mathbf{u}_{h}, \tilde{\mathbf{z}}_{h}\right)$ on the coarsest mesh is already very close to the reference $C_{1}$ value. Due to the high accuracy of the error estimates in Table 1 , we see that the error estimation significantly improves the computed force coefficients. In fact, while the adjointbased refinement reduces the number of degrees of freedom, which is required for meeting the accuracy tolerance, by a factor of 4 compared to global mesh refinement, there is another factor of about 16 gained by using the error estimation. Figures 2(d) and 2(f) show the respective plots for the error estimation and goal-oriented mesh that targets the drag and pitching moment coefficients, $C_{\mathrm{d}}$ and $C_{\mathrm{my}}$, respectively. Here, the behaviour is similar to that described before for the lift coefficient. In fact, here the enhanced force coefficients meet the accurarcy requirements on the second but coarsest mesh, which again corresponds to a very good error estimation.

Additionally, Figures 2(b), 2(d) and 2(f) show the convergence of the force coefficients on the sequence of multitarget refined meshes. Targeted at reducing the errors in $C_{\mathrm{l}}, C_{\mathrm{d}}$ and $C_{\mathrm{my}}$ simultaneously, the resulting values are not expected to be as accurate as for the single-target adapted meshes. However, we see that the differences for this test case are marginal. This demonstrates that by the multi-target mesh refinement about the same accuracy in the force coefficients is achieved for this test case as for the single-target mesh refinements (see also Figure 3). However, this is accomplished with significantly reduced computing. In fact, the multi-target approach requires only the flow solution, the adjoint solution and the solution to the discrete error equation on one sequence of adaptively refined meshes. In contrast to that, the single-target approach requires the flow solutions and the adjoint solutions on three different sequences of refined meshes. This results in a factor of 3 in the number of flow solutions and a factor of 1.5 in the number of auxiliary problems to be solved. Note, that these factors and thus the gain of using the multi-target approach increases with the number of target quantities under consideration. Finally, we note that the multi-target error estimation is comparable to the single-target error estimation since the enhanced $C_{\mathrm{d}}, C_{\mathrm{l}}$ and $C_{\mathrm{my}}$ values of (16) in Figures 2(b), 2(d) and 2(f) are close to the single-target enhanced values of (10).

In the following, we investigate the performance of the flow solver and of the solver for the additional discrete 


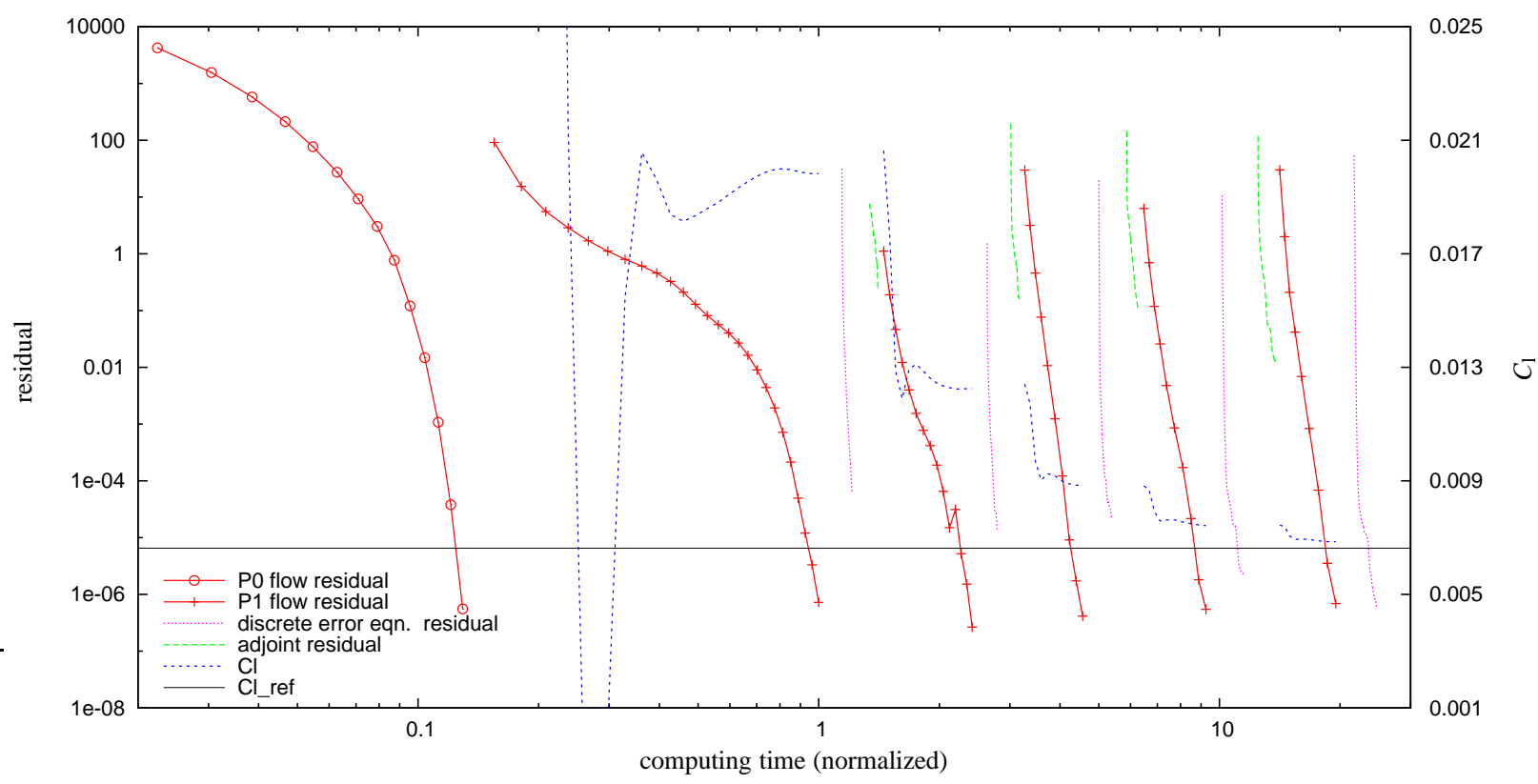

Figure 4: ADIGMA BTC0 test case at turbulent conditions: Convergence of the solver on the sequence of goal-oriented refined meshes that target the $C_{\mathrm{l}}, C_{\mathrm{d}}$ and $C_{\mathrm{m}}$ values: the nonlinear residuals and $C_{\mathrm{l}}$ values of the flow solutions and the linear residuals of the discrete error equations and the adjoint problems are shown.

error and adjoint equations. In particular, we are interested in the computing time required for solving the additional problems relative to the time spent for solving the flow problems in a multi-target adjoint-based mesh refinement algorithm. After initialization of freestream values on the coarsest mesh a flow solution $\mathbf{u}_{h} \in \mathbf{V}_{h, p}$ for $p=0$ is computed which serves as starting solution for $p=1$. On finer meshes the flow solution and the solutions to the discrete error and adjoint equations are initialized with interpolations of the solutions on the previous mesh. The nonlinear residual of the flow solution is decreased based on an implicit/backward Euler method where the CFL number and thus the local time step size is driven by the switched evolution relaxation (SER) method [42, 43]. In each nonlinear solution step a linear system is solved with the restarted and block-ILU-preconditioned GMRES method with 60 Krylov vectors. The linear solver is stopped once the linear residual is reduced by a factor of $10^{-6}$ or a maximum number of linear iterations is reached. On the coarsest mesh the linear solver performs at most 120 iterations; under mesh refinement this number increases with the cube root of the number of elements. The nonlinear solution process is stopped once the nonlinear residual is reduced to $10^{-6}$. Similarly, the linear problems arising from the discrete error equations and the adjoint problems are solved using 120 GMRES iteration steps with a restart after 60 iterations. Again, under mesh refinement the number of linear iteration steps increases with the cube root of the number of elements.

Figure 4 shows the convergence of the flow solver on the sequence of multi-target adjoint-based refined meshes that target the $C_{\mathrm{l}}, C_{\mathrm{d}}$ and $C_{\mathrm{m}}$ values, i.e. on the sequence of the 5 adaptively refined meshes considered in the right plots of Figure 2. In particular, Figure 4 shows the convergence of the nonlinear residuals and $C_{1}$ values of the flow solutions $\mathbf{u}_{h} \in \mathbf{V}_{h, 1}$. As the flow solution to this test case is particularly smooth, the flow solver takes very few iteration steps, only. In fact, the solver of the $p=1$ solution on the coarsest mesh requires 25 steps for convergence. This number decreases to less than 10 on subsequently refined meshes. Figure 4 also includes the convergence of the linear residuals of the solutions $\tilde{\mathbf{e}}_{h}$ and $\tilde{\mathbf{z}}_{h} \in \tilde{\mathbf{V}}_{h, 2}$ to the discrete error and adjoint equations. Although the discrete error equation is solved with a higher polynomial degree than the flow problems, it takes less computing time than the flow solver due to the linearity of the problem. In fact, approximating the discrete error on the coarsest mesh takes about $18 \%$ of the computing time of the flow solution. The additional computing time for the adjoint problem is of similar magnitude. We note that on finer meshes the computing time for the additional problems increases relative to the time taken by the flow solver due to the decreasing effort of the flow solver. However, already the additional $18 \%$ 


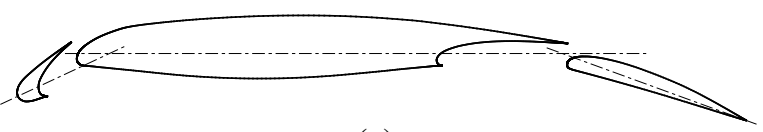

(a)

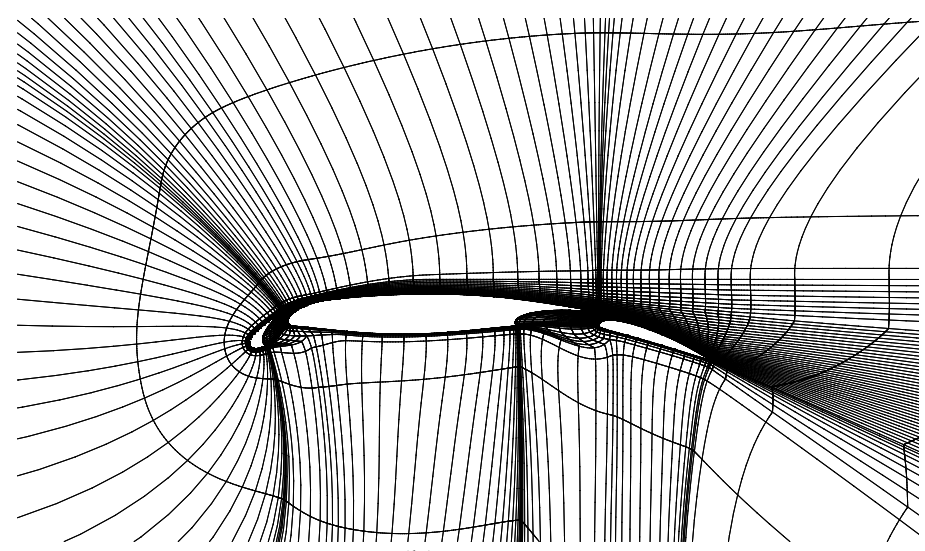

(b)

Figure 5: L1T2 high lift configuration: (a) Geometry of the L1T2 three-element airfoil. (b) Coarse grid of 4740 curved elements. The lines are given by polynomials of degree 4 .

computing time spent on the solution to the discrete error equation on top of the flow solver on the coarsest mesh yields an enhanced $C_{1}$ value that is within the prescribed accuracy tolerance (see Figure 2(b)). Furthermore, it results in significantly improved $C_{\mathrm{d}}$ and $C_{\mathrm{m}}$ values (see Figure 2(d)\&(f)). In contrast to that a higher-order flow solution $\mathbf{u}_{h} \in \mathbf{V}_{h, 2}$ on the coarsest mesh requires more than 5 times the time required for the $\mathbf{u}_{h} \in \mathbf{V}_{h, 1}$ flow solution.

\subsection{L1T2 high-lift configuration}

In this section we consider a turbulent flow around the L1T2 three-element airfoil (see Figure 5(a)) at a Mach number $M=0.197$, a Reynolds number $\operatorname{Re}=3.52 \cdot 10^{6}$ and an angle of attack $\alpha=20.18^{\circ}$. This case has been documented extensively in the literature (e.g., see [44, 45]). In particular, there are data of wind tunnel experiments available [46]. Based on extrapolation of higher order computational results and of the results on very fine locally adapted meshes, following reference values of the total drag and lift coefficients, $C_{\mathrm{d}}$ and $C_{1}$ have been obtained: $J_{C_{\mathrm{d}}}(\mathbf{u})=0.071$ and $J_{C_{1}}(\mathbf{u})=3.961$.

In the following the total drag and lift coefficients will be computed up to a predefined error tolerance TOL. Let us consider the following accuracy requirements:

$$
\begin{aligned}
\left|J_{C_{1}}(\mathbf{u})-J_{C_{1}}\left(\mathbf{u}_{h}\right)\right| \leq \mathrm{TOL}_{C_{1}} & =1 \cdot 10^{-2}, \\
\left|J_{C_{\mathrm{d}}}(\mathbf{u})-J_{C_{\mathrm{d}}}\left(\mathbf{u}_{h}\right)\right| \leq \mathrm{TOL}_{C_{\mathrm{d}}} & =5 \cdot 10^{-3} .
\end{aligned}
$$

Subsequently, we compare the performance in meeting these accuracy requirements for higher order discretizations against second-order discretizations on globally refined meshes. In addition, a comparison against the performance of the goal-oriented refinement approach that targets the $C_{1}$ and $C_{\mathrm{d}}$ coefficients is given.

An original block-structured mesh with 75840 elements has been agglomerated twice resulting in a coarse mesh of 4740 elements. As seen in Figure 5(b), the additional points of the original mesh have been used to define 4740 curved elements, where the curved lines are represented by polynomials of degree 4 .

On this mesh we first compute the flow solutions $\mathbf{u}_{h} \in \mathbf{V}_{h, p}$ for the polynomial degrees $p=1, \ldots, 4$. Additionally, for the lower polynomial degrees, we compute the solutions on globally refined meshes. The resulting force coefficients $C_{1}$ and $C_{\mathrm{d}}$ are given in Figures 6(a) and 6(c), respectively. In Figure 6(a) we see that the $p=3$ and 4 solutions are within the prescribed accuracy tolerance of the $C_{1}$ value on the coarsest mesh and are more accurate with significantly less degrees of freedom than the $p=1$ solution on the twice globally refined mesh. A similar behaviour is seen in Figure $6(\mathrm{c})$ for the $C_{\mathrm{d}}$ value. 


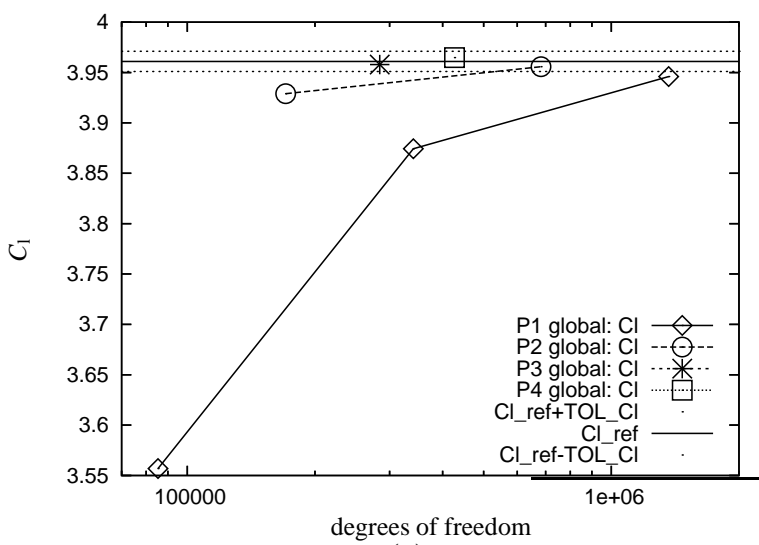

(a)

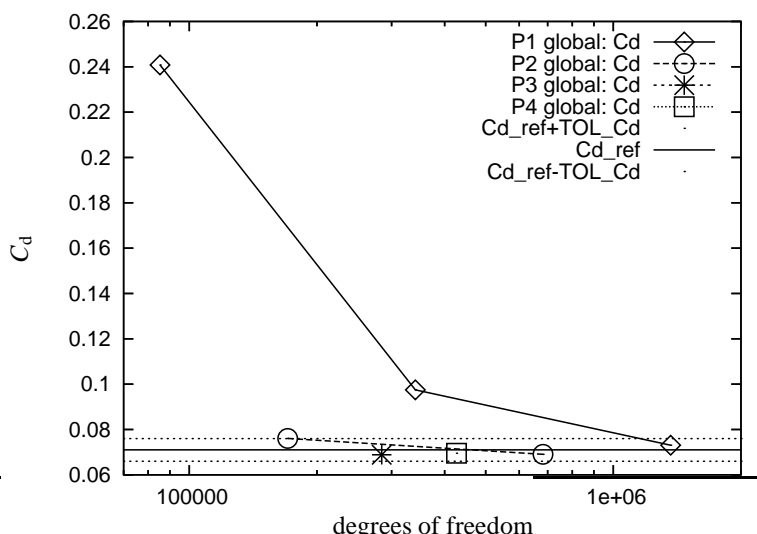

(c)

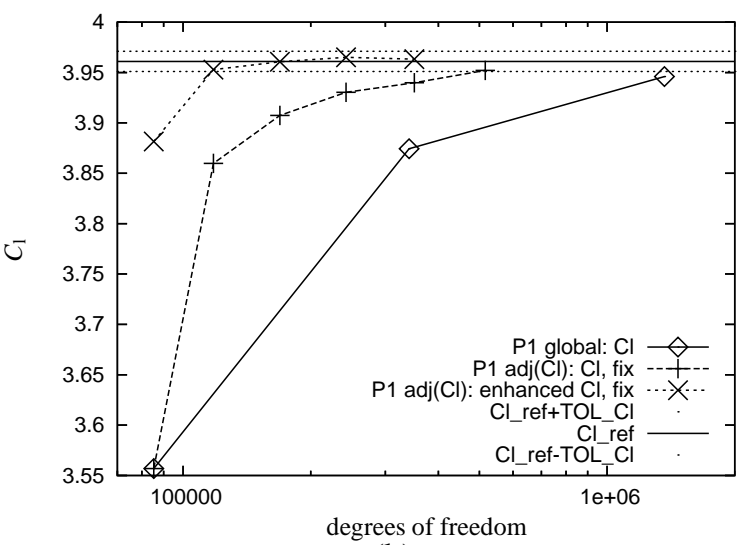

(b)

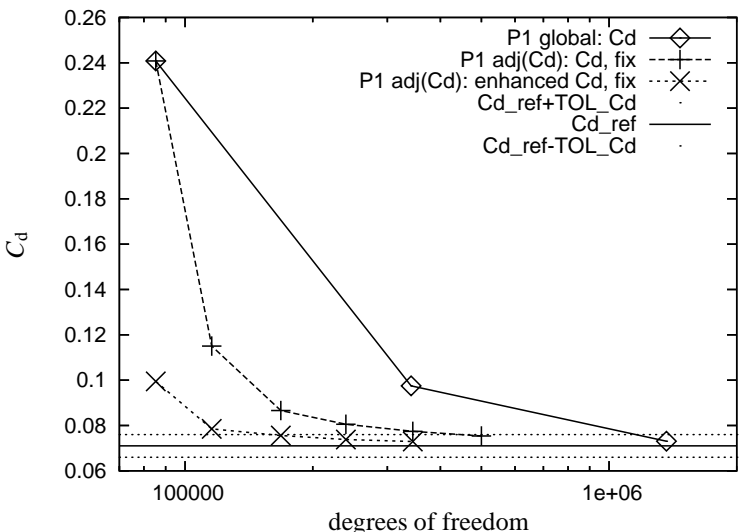

(d)

Figure 6: L1T2 high lift configuration: $C_{\mathrm{l}}$ and $C_{\mathrm{d}}$ values in the top and bottom row; (left) The $p=1, \ldots, 4$ solutions on globally refined meshes; (right) The force coefficients $J\left(\mathbf{u}_{h}\right)$ and the enhanced force coefficients (10) on adjoint-based refined meshes. 


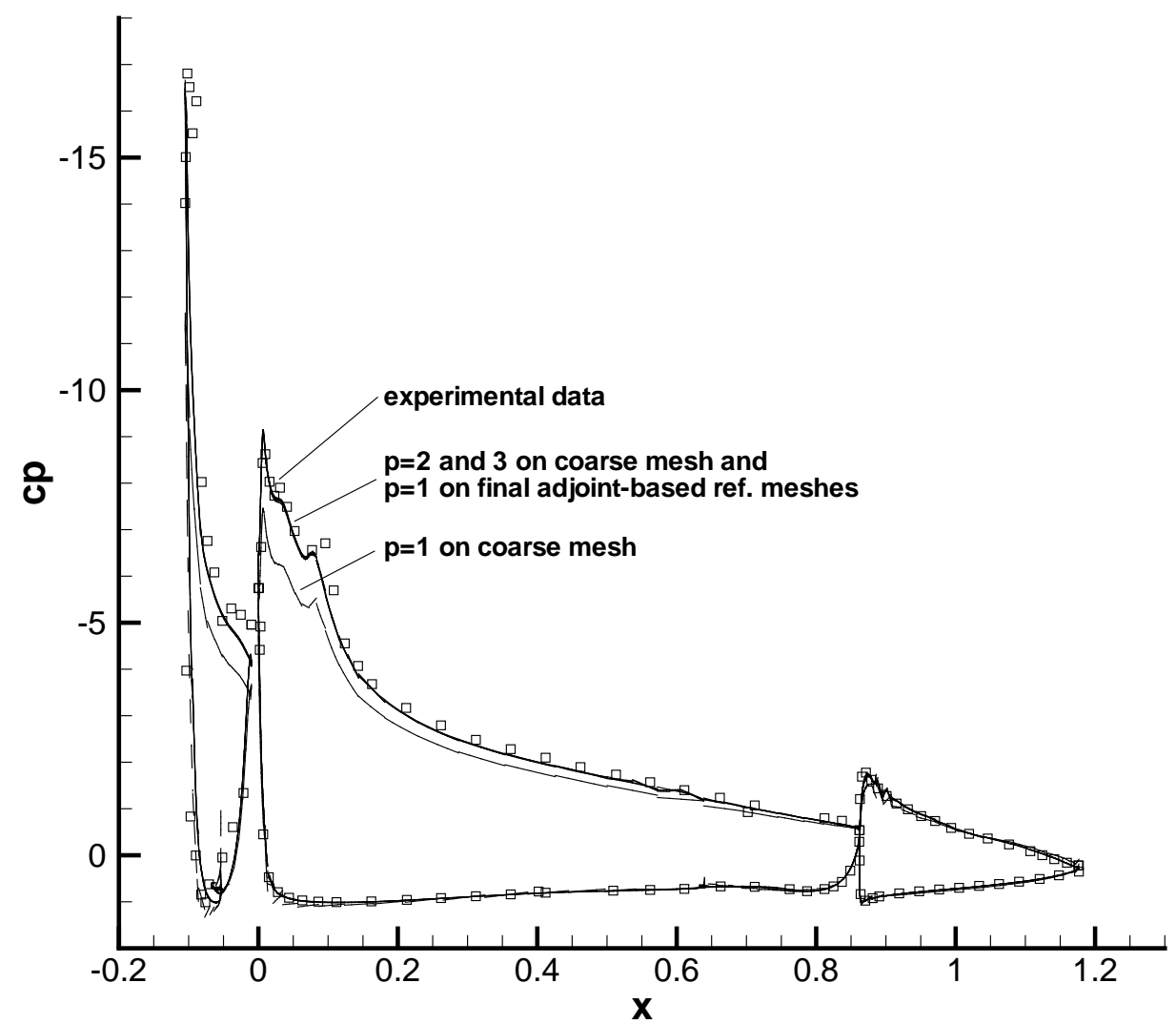

Figure 7: L1T2 high lift configuration: $c_{p}$ distribution of the $p=1,2$ and 3 solutions on the coarse mesh of 4740 curved elements compared to experimental data [46]. The $p=1$ solutions on the final $C_{\mathrm{l}}$ and $C_{\mathrm{d}}$ targeted adjoint-based refined meshes are also included. The single line is the $p=1$ solution on the coarse mesh; all other lines lie virtually on top of each other.

Let us now consider the adjoint-based error estimation and goal-oriented mesh refinement that targets the lift coefficient $C_{l}$. As in the previous example, we compute the solution $\mathbf{u}_{h} \in \mathbf{V}_{h, 1}$ to the flow problem (2), the solution $\tilde{\mathbf{z}}_{h} \in \mathbf{V}_{h, 2}$ to the discrete adjoint problem (8), evaluate the approximate error representation (9) and obtain the adjointbased indicators $\tilde{\eta}_{\kappa}$ that we employ for adaptive mesh refinement. Figure 6(b) shows the resulting $C_{1}$ values on the sequence of adaptively refined meshes. We see that the enhanced $C_{1}$ values of (10), which include the error estimation (7), reach the accuracy tolerance with significantly less degrees of freedom than the adjoint-based and global mesh refinement. In fact, after one refinement step the enhanced $C_{1}$ values are within the tolerance which corresponds to a very good error estimation. A similar behaviour can be seen in Figure $6(\mathrm{~d})$ for the $C_{\mathrm{d}}$ value. Here, we also see a good error estimation.

In Figure 7 a comparison is made between the $c_{p}$ distribution of the $p=1,2$, and 3 solutions on the coarse mesh of 4740 curved elements with the experimental data [46]. The $p=1$ solutions on the final $C_{1}$ and $C_{\mathrm{d}}$ targeted adjointbased refined meshes are also included in the comparison. Whereas there is a difference between the $p=1$ solution on the coarse mesh and the other solutions, there is virtually no difference visible for the other $c_{p}$ distributions which are in good agreement with the experiment. Figure 8 shows the respective comparison of the $c_{f}$ distributions. Here the jumps of the $p=2$ solution are smaller than in the $p=3$ solution (the $p=1$ solution is omitted). The $p=1$ solutions on the adjoint-based refined meshes are close together. However, despite the accuracy in the computed force coefficients neither of the $c_{f}$ distributions seem to be converged.

Finally, Figure 9 shows the adjoint-based refined meshes that target the $C_{1}$ and $C_{\mathrm{d}}$ values in comparison to the coarse mesh. We see that most of the refinement takes place in the vicinity of the airfoil. In both cases we see that the mesh has been refined in the neighborhood of the line which separates the recirculation zone behind the slat from the flow which passes between the slat and the main element. Additionally, the meshes have been refined in the neighborhood of the stagnation streamline of the main element. Although not clearly visible in the given plots, we note that there is refinement in the neighborhood of the stagnation lines of the slat and flap. Here, the adjoint solution 


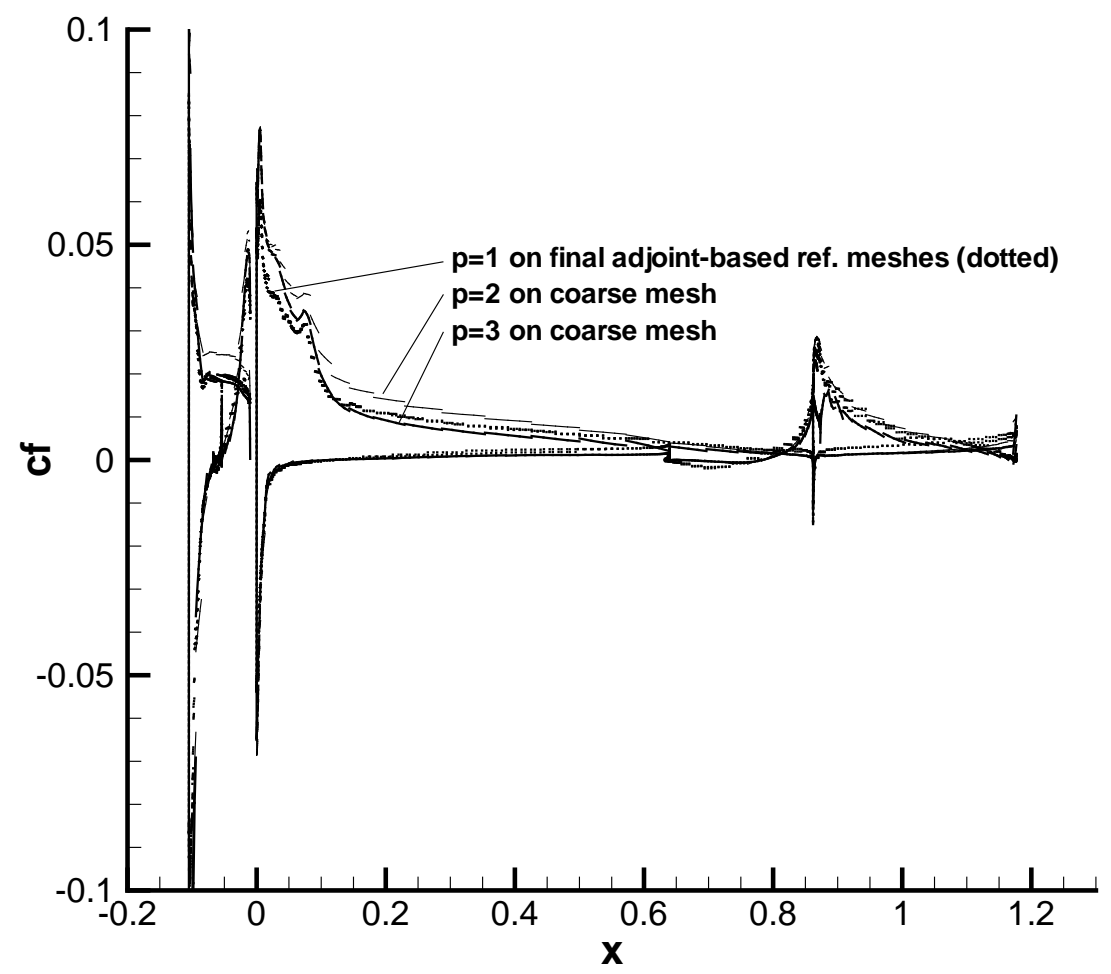

Figure 8: L1T2 high lift configuration: $c_{f}$ distribution of the $p=2$ and 3 solutions on the coarse mesh of 4740 curved elements (lines). The $p=1$ solutions on the final $C_{\mathrm{l}}$ and $C_{\mathrm{d}}$ targeted adjoint-based refined meshes are also included (dotted). The jumps of the $p=3$ solution are smaller than of the $p=2$ solution. The adjoint-based refined $p=1$ solutions are close together. 

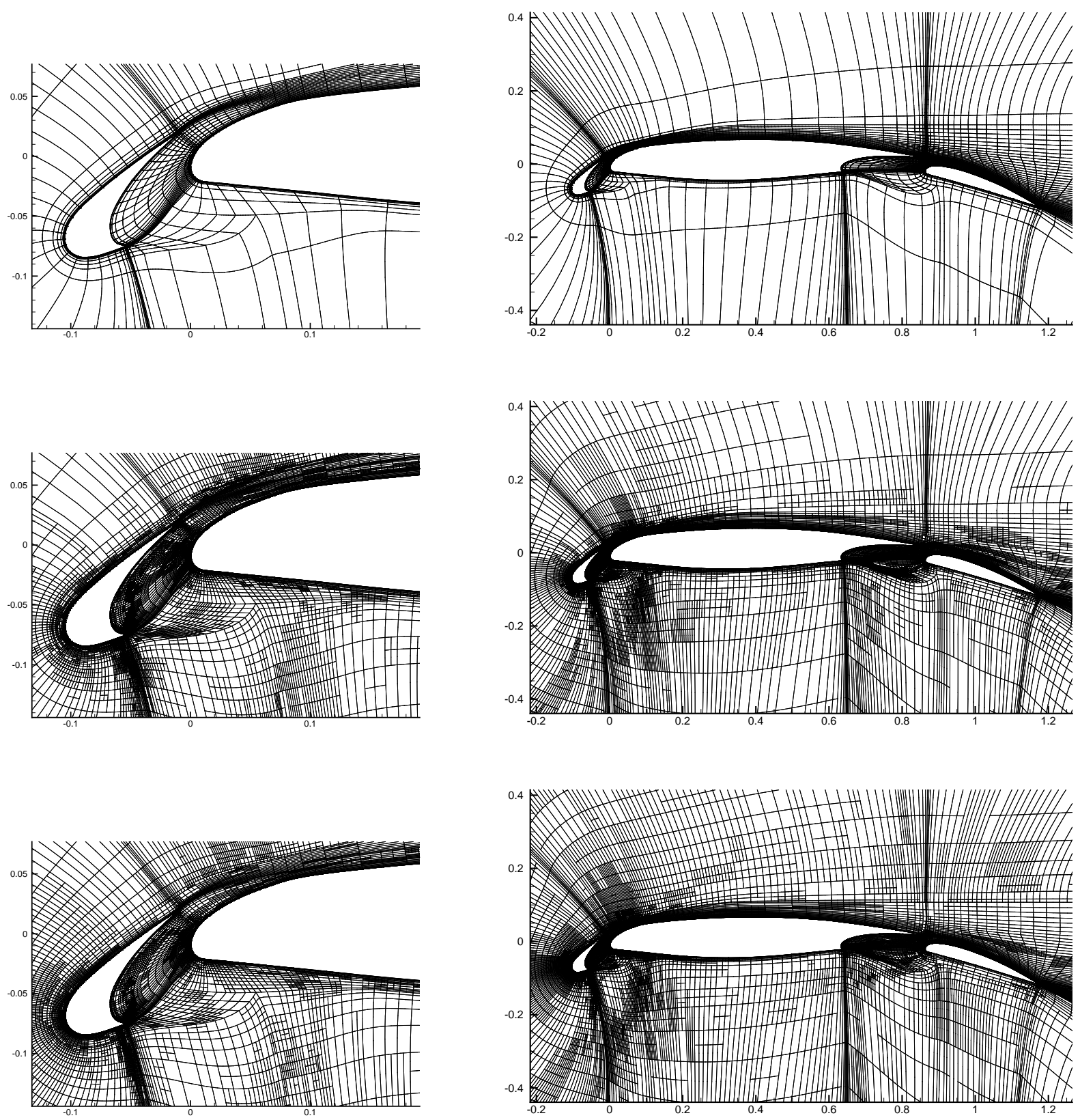

Figure 9: L1T2 high lift configuration: (top) Coarse mesh, and meshes after 5 adjoint-based mesh refinement steps that target (middle) $C_{1}$ and (bottom) $C_{\mathrm{d}}$. 


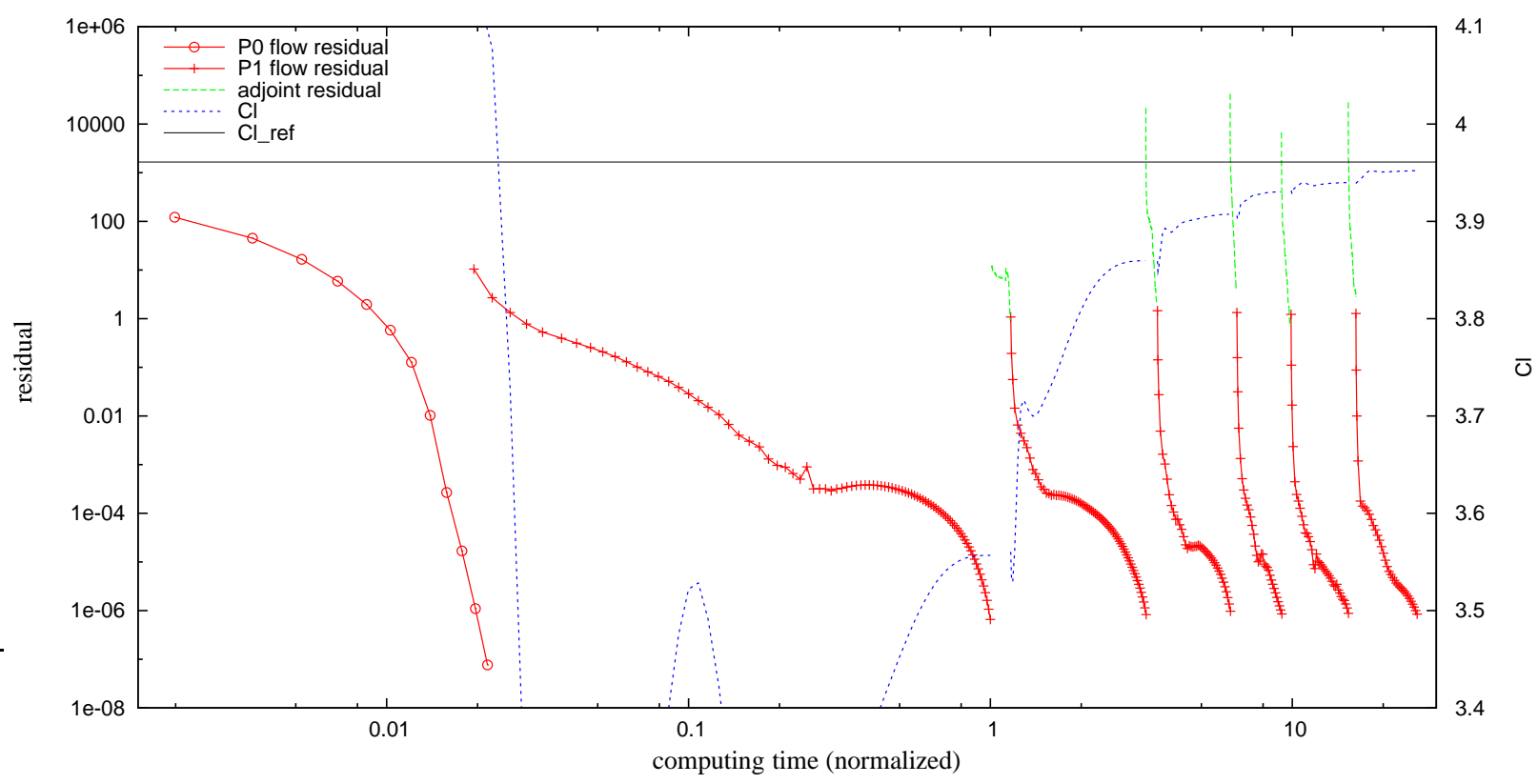

Figure 10: L1T2 high lift configuration: Convergence of the solver on the sequence of goal-oriented refined meshes that target the $C_{1}$ value: the nonlinear residuals and $C_{1}$ values of the flow solutions and the linear residuals of the adjoint solutions are shown. The adjoint solver takes about $12-16 \%$ of the time required for the flow solver.

indicates that the exact position of the stagnation points, as well as the flow upstream of them is particularly important for an accurate prediction of the aerodynamic force coefficients.

In the following, we investigate the performance of the flow solver and of the solver for the additional discrete error and adjoint equations. As in the previous test case, we are interested in the additional computing time required for solving the adjoint problems in an adjoint-based mesh refinement algorithm. The GMRES method applied to the linear problems arising in the implicit flow solver now uses at most 240 iterations on the coarsest mesh with a restart after 120 iterations. For the linear adjoint problems 960 iterations are used with a restart after every 240 iterations; under mesh refinement the maximum number of iterations increases with the square root of the number of elements.

Figure 10 shows the convergence of the flow and adjoint solvers on the sequence of adjoint-based refined meshes that target the $C_{1}$ value, i.e. on the sequence of the 6 adaptively refined meshes considered in Figure 6(b). In particular, Figure 10 shows the convergence of the nonlinear residual and $C_{1}$ value of the flow solutions $\mathbf{u}_{h} \in \mathbf{V}_{h, 1}$ as well as the convergence of the linear residual of the adjoint solutions $\tilde{\mathbf{z}}_{h} \in \mathbf{V}_{h, 2}$. First, we note that due to the complexity of the flow solution the flow solver applied to this high lift test case requires significantly more iteration steps than for the previous test case. In fact, Figure 10 shows that 90 solver steps are required for the $p=1$ solution on the coarsest mesh. This number reduces to about 35 on finer meshes. Although the adjoint problems are solved with a higher polynomial degree than the flow problems, the adjoint solver takes only a small fraction of the computional time of the flow solver. In fact, approximating the adjoint solutions takes between $12 \%$ and $16 \%$ of the computing time of the flow solutions on all meshes. This is due to the restricted number of linear iteration steps allowed in the adjoint solver. As we see in Figure 10, the linear residual is reduced by about 4 orders of magnitude only. Nevertheless, the resulting error estimation gives a significant improvement to the force coefficients as is seen in Figure 6(b). In contrast to that the solution $\mathbf{u}_{h} \in \mathbf{V}_{h, 2}$ to a higher order flow problem requires more than 4 times the time of the $\mathbf{u}_{h} \in \mathbf{V}_{h, 1}$ flow solution.

\subsection{Subsonic turbulent flow around the DLR-F6 wing-body configuration}

In this final example we consider a turbulent flow at a Mach number $M=0.5$, a Reynolds number $\operatorname{Re}=5 \cdot 10^{6}$ and an angle of attack $\alpha=-0.141$ around the DLR-F6 wing-body configuration without fairing (see Figure 11). This is a modification of the DPW III test case, where a fixed angle of attack has been assumed instead of a given target 


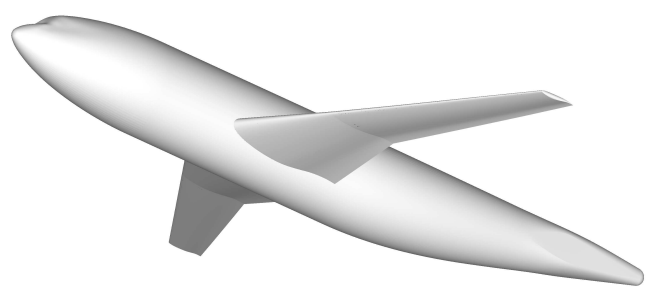

Figure 11: Geometry of the DLR-F6 wing-body configuration without fairing.

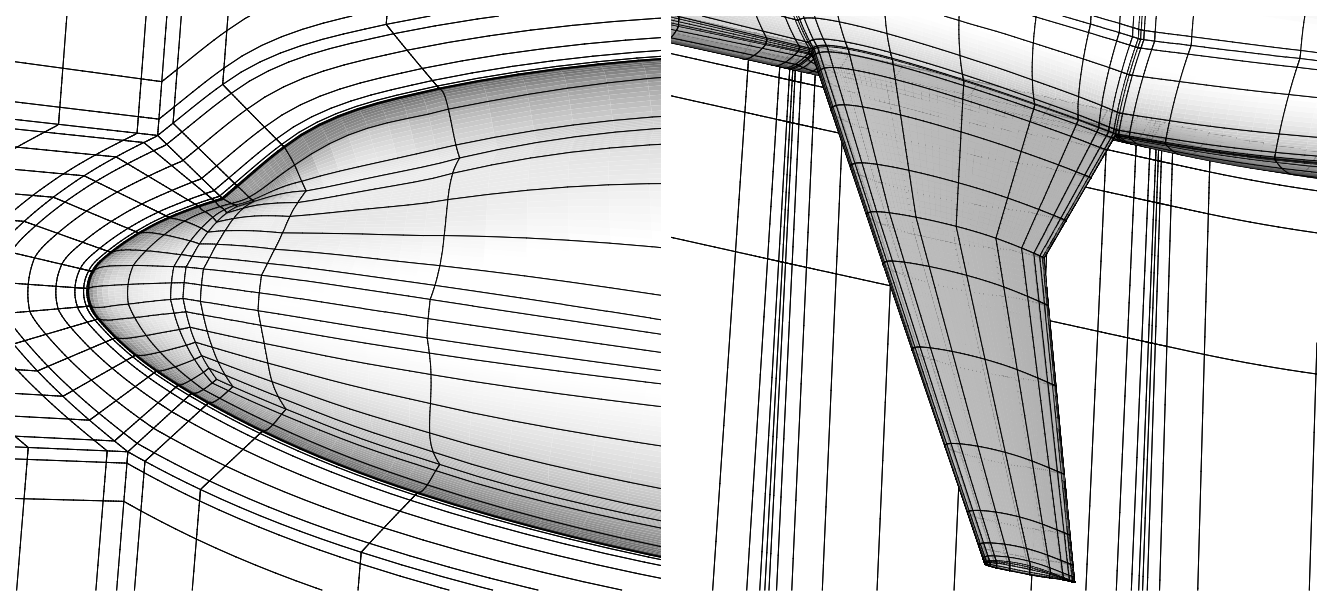

Figure 12: The DLR-F6 wing-body configuration: Mesh with 50618 curved elements shown close to the nose and the wing.

lift. Also, the Mach number has been reduced from the originally $M=0.75$ to $M=0.5$ in order to obtain a subsonic flow.

The original DPW mesh of about 3.2 million hexahedral elements has been agglomerated twice resulting in a coarse mesh of 50618 hexahedral elements. The additional points of the original mesh have been used to define 50618 curved elements (see Figure 12), where the curved lines are represented by polynomials of degree 4.

On this mesh we first compute the flow solutions $\mathbf{u}_{h} \in \mathbf{V}_{h, p}$ for the polynomial degrees $p=1,2$ and 3. Additionally, for $p=1$ and 2 we compute the solutions on a once globally refined mesh. The resulting drag coefficients are given in Figure 13. Due to the complexity of the problem, no rigorous convergence study and thus no reference value is available for this case. Nevertheless, we clearly see the advantage in terms of accuracy and degrees of freedom of using discretizations with higher polynomial degrees $p=2$ and 3 over the discretization with the low polynomial degree $p=1$.

Figure 14 shows the surface mesh near the wing-body junction, the $c_{p}$ distribution, and wall streamlines of the $p=1,2$ and 3 solutions on the coarse mesh. For the $p=2$ and $p=3$ solutions we clearly recognize the separation of the flow. The resolution of the $p=1$ solution on the coarse mesh is too low to capture the separation.

Let us now consider the adjoint-based error estimation and goal-oriented mesh refinement that targets the drag coefficient $C_{\mathrm{d}}$. As an example, Figure 15 shows the density adjoint (i.e., the first component of the discrete adjoint solution $\tilde{\mathbf{z}}_{h}$ ) on the locally adapted mesh after two adjoint-based mesh refinement steps. Finally, the $C_{\mathrm{d}}$ values and the enhanced $C_{\mathrm{d}}$ values on this sequence of adjoint-based refined meshes are given in Figure 13. As in the previous examples, we see here a significant decrease in the number of degrees of freedom required for computing the force coefficient up to a specific accuracy. A further significant improvement can be seen in the enhanced force coefficient, which again corresponds to a good error estimation.

As already seen in Figure 14(b) the $p=1$ solution on the coarse mesh does not capture the separation. Without showing details, we note that the $p=1$ solution on the twice adjoint-based refined mesh has an onset of separation which is then fully captured on the three times adjoint-based refined mesh. 


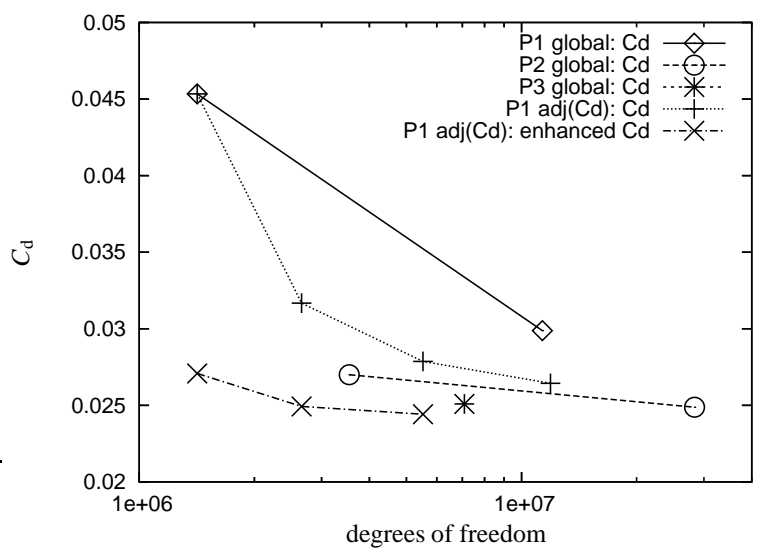

Figure 13: Turbulent flow around the DLR-F6 wing-body configuration: Convergence of the drag coefficient $C_{\mathrm{d}}$ for the $p=1,2$ and 3 solutions on the coarse and a once globally refined mesh. Convergence of the $C_{\mathrm{d}}$ and the enhanced $C_{\mathrm{d}}$ values under adjoint-based mesh refinement targeted at the $C_{\mathrm{d}}$ value.

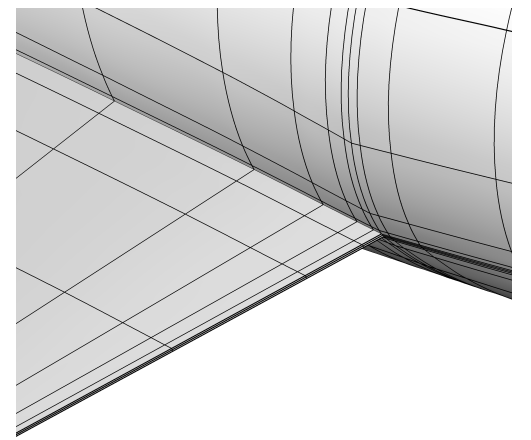

(a)

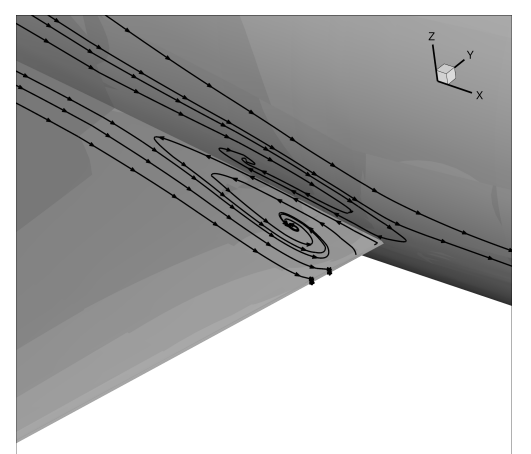

(c)

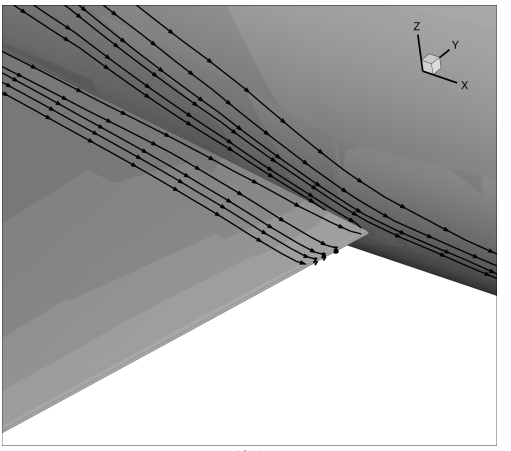

(b)

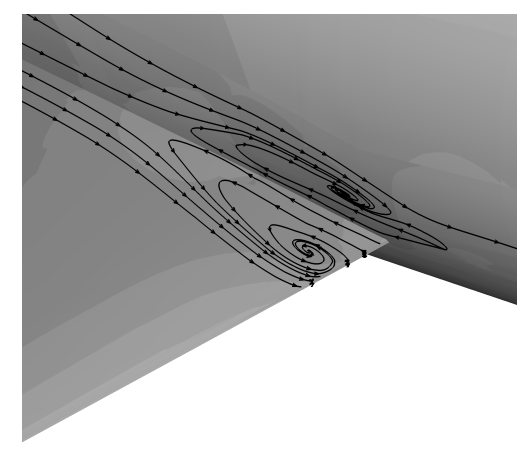

(d)

Figure 14: Turbulent flow around the DLR-F6 wing-body configuration: (b)-(d) $c_{p}$ distributions and wall streamlines of the $p=1,2$ and 3 solutions on (a) the coarse mesh of 50618 curved elements. 

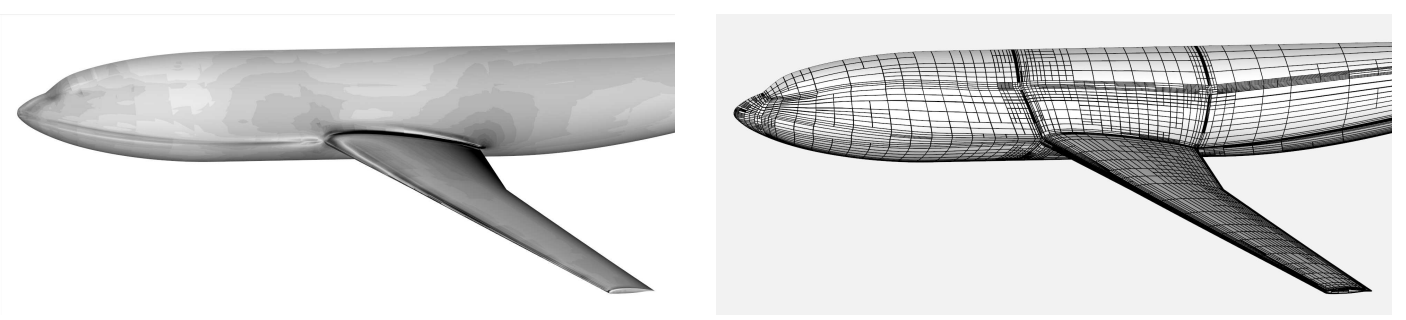

Figure 15: Turbulent flow around the DLR-F6 wing-body configuration: Density adjoint distribution, i.e., the distribution of the first component of the discrete adjoint solution $\tilde{\mathbf{z}}_{h}$, on a twice adjoint-based refined mesh.

\section{Conclusions}

In this article we applied higher order and adaptive discontinuous Galerkin methods to turbulent aerodynamic test cases as governed by the RANS- $k \omega$ equations. In particular, we considered three test cases of increasing complexity, including turbulent flows around a three-dimensional streamlined body, the three-element L1T2 high lift configuration and the DLR-F6 wing body configuration.

For each of the test cases we showed that DG discretizations of higher polynomial degrees are advantageous in terms of accuracy and number of degrees of freedom over discretizations of lower polynomial degrees. Furthermore, we showed that using adjoint-based refinement specific accuracy requirements on the aerodynamic force coefficients were met with a significantly reduced number of degrees of freedom. Adjoint-based error estimation has been shown to give accurate and reliable error estimates for each of the test cases. In particular, it was shown that using the error estimates the force coefficients can be significantly improved. Finally, it was demonstrated that the multi-target error estimation and adjoint-based mesh refinement approach can successfully be applied to $3 \mathrm{~d}$ turbulent flows. In fact, for the case considered, the force coefficients and the error estimation on the multi-target refined meshes was comparable in accuracy to the single-target adapted meshes with a significantly reduced computational effort.

In this work the effect of using higher order discretizations on the one hand and adjoint-based error estimation and mesh refinement approaches on the other hand have been considered separately. Ideally, the computational mesh and the polynomial degree should be adapted simultaneously, leading to so-called $h p$-adaptive methods. While $h p$ refinement in the context of compressible flows has been successfully applied to $2 \mathrm{~d}$ inviscid and laminar flows [8, 47], a significant research effort is required for transferring these approaches to $3 \mathrm{~d}$ turbulent flows, in particular, with respect to the required stability of the turbulent flow solver on $h p$-refined meshes.

\section{Acknowledgments}

The authors acknowledge the partial financial support of both the President's Initiative and Networking Fund of the Helmholtz Association of German Research Centres and the European project ADIGMA [48]. Computations have been performed using the DG flow solver PADGE [49] which is based on a modified version of the deal. II library [50].

\section{References}

[1] F. Bassi, A. Crivellini, S. Rebay, M. Savini, Discontinuous Galerkin solution of the Reynolds-averaged Navier-Stokes and $k-\omega$ turbulence model equations, Computers \& Fluids 34 (2005) 507-540.

[2] F. Bassi, A. Crivellini, A. Ghidoni, S. Rebay, High-order discontinuous Galerkin discretization of transonic turbulent flows, 47th AIAA Aerospace Sciences Meeting, 2009. AIAA 2009-180.

[3] K. J. Fidkowski, D. L. Darmofal, A triangular cut-cell adaptive method for high-order discretizations of the compressible Navier-Stokes equations, J. Comput. Physics 225 (2007) 1653-1672.

[4] K. J. Fidkowski, T. A. Oliver, J. Lu, D. L. Darmofal, p-multigrid solution of high-order discontinuous Galerkin discretizations of the compressible Navier-Stokes equations, J. Comput. Phys. 207 (2005) 92-113.

[5] H. Luo, J. D. Baum, R. Löhner, A p-multigrid discontinuous Galerkin method for the Euler equations on unstructured grids, J. Comput. Phys. 211 (2006).

[6] P.-O. Persson, J. Peraire, Sub-cell shock capturing for discontinuous Galerkin methods, $44^{\text {th }}$ AIAA Aerospace Sciences Meeting and Exhibit, 2006. AIAA 2006-112. 
[7] B. Landmann, M. Kessler, S. Wagner, E. Krämer, A parallel discontinuous Galerkin code for the Navier-Stokes equations, $44^{\text {th }}$ AIAA Aerospace Sciences Meeting and Exhibit, 2006. AIAA 2006-111.

[8] L. Wang, D. J. Mavriplis, Adjoint-based $h$ - $p$ adaptive discontinuous Galerkin methods for the 2d compressible Euler equations, J. Comput. Phys. 228 (2009) 7643-7661.

[9] C. Baumann, J. Oden, A discontinuous $h p$ finite element method for the Euler and Navier-Stokes equations, Int. J. Numer. Meth. Fluids 31 (1999) 79-95.

[10] C. R. Nastase, D. J. Mavriplis, High-order discontinuous Galerkin methods using an hp-multigrid approach, J. Comput. Phys. 213 (2006) 330-357.

[11] N. Kroll, ADIGMA - A European project on the development of adaptive higher-order variational methods for aerospace applications, 47th AIAA Aerospace Sciences Meeting, 2009. AIAA 2009-176.

[12] R. Becker, R. Rannacher, A feed-back approach to error control in finite element methods: Basic analysis and examples, East-West J. Numer. Math. 4 (1996) 237-264.

[13] R. Becker, R. Rannacher, An optimal control approach to a posteriori error estimation in finite element methods, Acta Numerica 10 (2001) $1-102$.

[14] R. Hartmann, Adaptive Finite Element Methods for the Compressible Euler Equations, Ph.D. thesis, University of Heidelberg, 2002.

[15] R. Hartmann, P. Houston, Adaptive discontinuous Galerkin finite element methods for the compressible Euler equations, J. Comput. Phys. 183 (2002) 508-532.

[16] J. Lu, An a posteriori Error Control Framework for Adaptive Precision Optimization using Discontinuous Galerkin Finite Element Method, Ph.D. thesis, M.I.T., 2005.

[17] R. Hartmann, P. Houston, Symmetric interior penalty DG methods for the compressible Navier-Stokes equations II: Goal-oriented a posteriori error estimation, Int. J. Num. Anal. Model. 3 (2006) 141-162.

[18] R. Hartmann, Adaptive discontinuous Galerkin methods with shock-capturing for the compressible Navier-Stokes equations, Int. J. Numer. Meth. Fluids 51 (2006) 1131-1156.

[19] D. A. Venditti, D. L. Darmofal, Grid adaptation for functional outputs: application to two-dimensional inviscid flows, J. Comp. Phys. 176 (2002) 40-69.

[20] T. Leicht, Anisotropic mesh refinement for discontinuous Galerkin methods in aerodynamic flow simulations, Diploma thesis, Dresden University of Technology, 2006.

[21] T. Leicht, R. Hartmann, Anisotropic mesh refinement for discontinuous Galerkin methods in two-dimensional aerodynamic flow simulations, Int. J. Numer. Meth. Fluids 56 (2008) 2111-2138.

[22] T. Leicht, R. Hartmann, Error estimation and anisotropic mesh refinement for 3d laminar aerodynamic flow simulations, J. Comput. Phys. 229 (2010) 7344-7360.

[23] T. A. Oliver, A High-Order, Adaptive, Discontinuous Galerkin Finite Element Method for the Reynolds-Averaged Navier-Stokes Equations, Ph.D. thesis, Department of Aeronautics \& Astronautics, Massachusetts Institute of Technology, 2008.

[24] L. Formaggia, S. Micheletti, S. Perotto, Anisotropic mesh adaptation in computational fluid dynamics: Application to the advection-diffusionreaction and the Stokes problems, Applied Numerical Mathematics 51 (2004) 511-533.

[25] S. Micheletti, S. Perotto, Output functional control for nonlinear equations driven by anisotropic mesh adaption. The Navier-Stokes equations, SIAM J. Sci. Comput. 30 (2008) 2817-2854.

[26] D. A. Venditti, D. L. Darmofal, Anisotropic grid adaptation for functional outputs: application to two-dimensional viscous flows, J. Comp. Phys. 187 (2003) 22-46.

[27] R. Hartmann, P. Houston, Goal-oriented a posteriori error estimation for multiple target functionals, in: T. Y. Hou, E. Tadmor (Eds.), Hyperbolic problems: theory, numerics, applications, Springer, 2003, pp. 579-588.

[28] R. Hartmann, Multitarget error estimation and adaptivity in aerodynamic flow simulations, SIAM J. Sci. Comput. 31 (2008) $708-731$.

[29] M. Nemec, M. J. Aftosmis, Error estimation and adaptive refinement for embedded-boundary Cartesian meshes, 45th AIAA Aerospace Sciences Meeting, 2007. AIAA Paper 2007-4187.

[30] M. Nemec, M. J. Aftosmis, M. Wintzer, Adjoint-based adaptive mesh refinement for complex geometries, 46th AIAA Aerospace Sciences Meeting, 2008. AIAA Paper 2008-0725.

[31] D. C. Wilcox, Reassessment of the scale-determining equation for advanced turbulence models, AIAA J. 26 (1988) 1299-1310. 1988.

[32] D. C. Wilcox, Turbulence Modeling for CFD, DCW Industries, Inc., La Canada CA, 1993.

[33] F. Ilinca, D. Pelletier, Positivity preservation and adaptive solution for the $k-\epsilon$ model of turbulence, AIAA J. 36 (1998) $44-50$.

[34] F. Bassi, S. Rebay, A high order discontinuous Galerkin method for compressible turbulent flows, in: B. Cockburn, G. Karniadakis, C.-W. Shu (Eds.), Discontinuous Galerkin Methods, volume 11 of Lecture Notes in Comput. Sci. Engrg., Springer, 2000 , pp. 77-79.

[35] F. Bassi, S. Rebay, GMRES discontinuous Galerkin solution of the compressible Navier-Stokes equations, in: B. Cockburn, G. Karniadakis, C.-W. Shu (Eds.), Discontinuous Galerkin Methods, volume 11 of Lecture Notes in Comput. Sci. Engrg., Springer, 2000, pp. 197-208.

[36] F. Bassi, S. Rebay, Numerical evaluation of two discontinuous Galerkin methods for the compressible Navier-Stokes equations, Int. J. Numer. Meth. Fluids 40 (2002) 197-207.

[37] R. Hartmann, P. Houston, An optimal order interior penalty discontinuous Galerkin discretization of the compressible Navier-Stokes equations, J. Comput. Phys. 227 (2008) 9670-9685.

[38] R. Hartmann, Adjoint consistency analysis of discontinuous Galerkin discretizations, SIAM J. Numer. Anal. 45 (2007) 2671-2696.

[39] G. Karniadakis, S. Sherwin, Spectral/hp Finite Element Methods in CFD, Oxford University Press, 1999.

[40] P. Šolín, K. Segeth, I. Doležel, Higher-Order Finite Element Methods, Chapman \& Hall/CRC, 2004.

[41] F. R. Menter, Two equation eddy-viscosity turbulence models for engineering applications, AIAA J. 32 (1994) $1598-1605$.

[42] W. A. Mulder, B. van Leer, Experiments with implicit upwind methods for the Euler equations, J. Comput. Phys. 59 (1985) 232-246.

[43] J. A. Essers, M. Delanaye, P. Rogiest, Upwind-biased finite volume technique solving Navier-Stokes equations on irregular meshes, AIAA Journal 33 (1995).

[44] I. Fejtek, Summary of code validation results for a multiple element airfoil test case, 28th AIAA fluid dynamics conference, 1997. AIAA 
Paper 97-1932.

[45] A. Hellsten, New Two-Equation Turbulence Model for Aerodynamics Applications, Technical Report Report No. A-21, Helsinki University of Technology, Laboratory of Aerodynamics, 2004.

[46] I. R. M. Moir, Measurements on a two-dimensional aerofoil with high-lift devices, AGARD Advisory Report 303, Advisory Group for Aerospace Research \& Development, Neuilly-sur-Seine, 1994. Test case A2.

[47] R. Hartmann, P. Houston, Error estimation and adaptive mesh refinement for aerodynamic flows, in: H. Deconinck (Ed.), VKI LS 2010-01: $36^{\text {th }}$ CFD/ADIGMA course on hp-adaptive and hp-multigrid methods, Oct. 26-30, 2009, Von Karman Institute for Fluid Dynamics, Rhode Saint Genèse, Belgium, 2010.

[48] N. Kroll, Adgima - a European project on the development of adaptive higher-order variational methods for aerospace applications, 47th AIAA Aerospace Sciences Meeting, 2009. AIAA 2009-176.

[49] R. Hartmann, J. Held, T. Leicht, F. Prill, Discontinuous Galerkin methods for computational aerodynamics - 3D adaptive flow simulation with the DLR PADGE code, Aerospace Science and Technology 14 (2010) 512-519.

[50] W. Bangerth, R. Hartmann, G. Kanschat, deal.II - A general purpose object oriented finite element library, ACM Transactions on Mathematical Software 33 (2007) 24:1-24:27. 